\title{
Different Subthreshold Mechanisms Underlie Song Selectivity in Identified HVc Neurons of the Zebra Finch
}

\author{
Richard Mooney \\ Department of Neurobiology, Duke University Medical Center, Durham, North Carolina 27710
}

\begin{abstract}
Songbirds learn and maintain their songs via auditory experience. Neurons in many telencephalic nuclei important to song production and development are song selective, firing more to forward auditory playback of the bird's own song (BOS) than to reverse BOS or conspecific songs. Elucidating circuits that generate these responses can localize where auditory experience influences vocalization, bridging cellular and systems analyses of song learning. Song-selective responses in many song nuclei, including the vocal premotor nucleus robustus archistriatalis (RA) and the basal ganglia homolog area $X$, are thought to originate in nucleus HVc (used as a proper name), which contains interneurons and relay cells that innervate either RA or area X. Previous studies indicated that only $\mathrm{X}$-projecting neurons have auditory responses, leaving open the source of RA's auditory input and the degree to which song selectivity may be refined in HVc. Here, in vivo intracellular recordings from morphologically and electrophysiologically identified HVc neurons revealed that both relay
\end{abstract}

cell types fire song-selectively. However, their firing arises via markedly different subthreshold processes, and only X-projecting neurons appear to be sites for auditory refinement. RA-projecting neurons exhibited purely depolarizing subthreshold responses that were highly song selective and that were excitatory. In contrast, subthreshold responses of X-projecting neurons included less-selective depolarizing and highly selective hyperpolarizing components. Within individual birds, these BOS-evoked hyperpolarizations closely matched interneuronal firing, suggesting that $\mathrm{HVc}$ interneurons make restricted inputs onto X-projecting neurons. Because of the two relay cell types' subthreshold differences, factors affecting their resting membrane potentials could enable them to transmit distinct song representations to their targets.

Key words: HVc; in vivo intracellular; vocal learning; songbirds; song; auditory selectivity; communication; zebra finch
Songbirds and humans listen to their own vocalizations to develop and maintain learned communication sounds (Doupe and Kuhl, 1999). These processes are likely to require the integration of auditory and vocal circuitry. Indeed, in songbirds, neurons in many brain nuclei used for song production, development, and perception are song selective, firing more to forward auditory playback of the bird's own song (BOS) than to reverse BOS or conspecific songs (Margoliash, 1983; Doupe and Konishi, 1991; Janata and Margoliash, 1999). Elucidating the circuitry underlying song selectivity could reveal where audition influences vocalization, thus providing a cellular framework for vocal learning. In fact, auditory responses in many song-related nuclei may arise from two distinct relay cell classes in the nucleus $\mathrm{HVc}$ (used as a proper name), but the correspondence between auditory responsiveness and $\mathrm{HVc}$ cell type is poorly understood. This study uses in vivo intracellular recordings from identified $\mathrm{HVc}$ neurons to establish this correspondence and to probe the cellular mechanisms underlying song selectivity.

The telencephalic nucleus $\mathrm{HVc}$ is a major auditory-vocal interface. HVc exhibits singing-related premotor activity (McCasland, 1987; Yu and Margoliash, 1996), and many HVc neurons are song selective (Margoliash, 1986; Lewicki and Arthur, 1996; Volman, 1996; Theunissen and Doupe, 1998). Beyond affording a site for auditory-vocal integration, $\mathrm{HVc}$ is the probable source of auditory input to other nuclei important to song production, development, and perception. $\mathrm{HVc}$ is structurally heterogeneous, containing two relay cell types and interneurons, and functionally heterogeneous,

\footnotetext{
Received Feb. 7, 2000; revised April 27, 2000; accepted May 5, 2000.

This work was supported by National Institutes of Health Grant R01 DC 02524 and by grants from the McKnight, Sloan, and Klingenstein Foundations. I am grateful for helpful discussions and comments on this manuscript provided by Dr. David Fitzpatrick, Dr. John Spiro, Merri Rosen, and other members of the Mooney lab, as well as two anonymous reviewers. Special thanks to Stacey James for expert histology, Jim Adelman for data analysis, and Merri Rosen for expert computer programming.

Correspondence should be addressed to Dr. Richard Mooney, Department of Neurobiology, Box 3209, Duke University Medical Center, Durham, NC 27710. E-mail: mooney@neuro.duke.edu.

Copyright (C) 2000 Society for Neuroscience $0270-6474 / 00 / 205420-17 \$ 15.00 / 0$
}

because its two relay cell types give rise to anatomically separate pathways implicated in either audition-dependent vocal plasticity or vocal control (see Fig. 2) (Nottebohm et al., 1976; Fortune and Margoliash, 1995; Foster and Bottjer, 1998). One relay cell type innervates area $\mathrm{X}$, a basal ganglia homolog within the anterior forebrain pathway (AFP). The AFP, which is essential to juvenile and adult audition-dependent vocal plasticity (Bottjer et al., 1984; Scharff and Nottebohm, 1991; Brainard and Doupe, 2000) and may facilitate song perception (Scharff et al., 1998) is thought to derive auditory input from HVc (Vates et al., 1996). In agreement with this idea, in vivo intracellular recordings from $\mathrm{X}$-projecting neurons reveal that they have song-selective auditory responses (Lewicki, 1996). Furthermore, certain X-projecting neurons display BOSevoked hyperpolarizations (Lewicki, 1996), perhaps driven by $\mathrm{HVc}$ interneurons, that might refine these relay cells' song selectivity relative to HVc's auditory afferents (Lewicki and Arthur, 1996). However, the auditory properties of HVc interneurons and their interactions with either relay cell type are unknown. The second relay cell type innervates the nucleus robustus archistriatalis (RA), which controls the vocal and respiratory neurons used for singing (Vicario, 1993; Wild, 1993). Although RA has auditory responses requiring direct HVc input (Doupe and Konishi, 1991; Vicario and Yohay, 1993) and RA-projecting neurons afford attractive sites for potential auditory-vocal interaction, previous intracellular studies concluded they were nonauditory (Katz and Gurney, 1981; Lewicki, 1996). The difficulty in obtaining in vivo intracellular recordings from identified $\mathrm{HVc}$ neurons has obscured the nature of auditory information transmitted from $\mathrm{HVc}$ to other song nuclei, while impeding insight into the mechanisms underlying song selectivity.

Here, in vivo intracellular recordings from identified $\mathrm{HVc}$ neurons were used to measure their responses to BOS playback. These studies show that HVc's two relay cell populations generate similar song-selective firing via distinct subthreshold processes, which may arise via segregated input from highly selective interneurons onto $\mathrm{X}$-projecting cells. Ultimately, factors affecting the two relay cell types' membrane potentials may unmask previously unidentified 
Table 1. In vivo electrophysiological properties of morphologically identified HVc neurons

\begin{tabular}{|c|c|c|c|c|c|c|c|}
\hline Cell type $(n) *$ & $\begin{array}{l}\text { Resting po- } \\
\text { tential, mV }\end{array}$ & $\mathrm{R}_{\text {input }}, \mathrm{M} \Omega$ & $\begin{array}{l}\text { Spontaneous } \\
\text { firing rate, } \mathrm{Hz}\end{array}$ & $\begin{array}{l}\text { Evoked firing } \\
\text { rate, } \mathrm{Hz} / \mathrm{nA}\end{array}$ & $\begin{array}{l}\text { Spike width, } \\
\text { msec }\end{array}$ & $\mathrm{AHP}, \mathrm{mV}$ & $\begin{array}{l}\text { Latency to first } \\
\text { spike, msec (number } \\
\text { of spike onsets; } \\
+400 \text { and }+600 \text { pA) }\end{array}$ \\
\hline X-projecting (16) & $-76 \pm 2$ & $48 \pm 6$ & $1.5 \pm 0.4$ & $42 \pm 2$ & $2.0 \pm 0.2$ & $14.2 \pm 0.6$ & $28 \pm 10(40)$ \\
\hline RA-projecting (10) & $-78 \pm 3$ & $50 \pm 10(9)$ & $0.6 \pm 0.4$ & $24 \pm 6(9)$ & $1.8 \pm 0.4(9)$ & $13.4 \pm 1.9(9)$ & $\begin{array}{l}\mathbf{1 7 2} \pm \mathbf{6 7}(14) \\
p<0.001, \mathrm{Xp} p< \\
\quad 0.01, \text { Int two- } \\
\quad \text { tailed } t \text { test }\end{array}$ \\
\hline Interneuron (11) & $-66 \pm 2$ & $44 \pm 6$ & $\begin{array}{l}\mathbf{1 2 . 0} \pm \mathbf{4 . 3} * *(6) \\
p<0.01 \mathrm{vs} \\
\text { RAp,Xp } \\
\text { two-tailed } \\
t \text { test }\end{array}$ & $\begin{array}{l}\mathbf{1 7 2} \pm \mathbf{2 2} \\
p<0.0001, \\
\mathrm{RAp}, \mathrm{Xp}, \\
\text { two-tailed } t \\
\text { test }\end{array}$ & $\begin{array}{c}\mathbf{0 . 7} \pm \mathbf{0 . 0 6} \\
p<0.01 \text { vs } \\
\text { RAp, } p< \\
0.0001 \text { vs } \\
\text { Xp, two } \\
\text { tailed } t \text { test }\end{array}$ & $\begin{array}{c}\mathbf{1 7 . 6} \pm \mathbf{0 . 6} \\
p<0.05 \mathrm{RAp} \\
p<0.001 \\
\mathrm{Xp}, \text { two- } \\
\text { tailed } t \text { test }\end{array}$ & $15 \pm 9$ \\
\hline
\end{tabular}

*Numbers in this column apply to all other columns, except as noted in parentheses. **Values in bold are significantly different from similar values in the other cell types.

subthreshold differences, enabling distinct auditory representations of song to be transmitted by HVc.

\section{MATERIALS AND METHODS}

Subjects. Experiments were performed using 31 adult [85-440 posthatch days (PHD)] male zebra finches (Taeniopygia guttata) in accordance with a protocol approved by the Duke University Institutional Animal Care and Use Committee.

Stimuli. Before the experiment, songs were recorded from a male zebra finch placed with an adult female zebra finch in a recording chamber (Industrial Acoustics, Bronx, NY). Songs were amplified and low-pass filtered at $10 \mathrm{kHz}$, digitized at $20 \mathrm{kHz}$ (National Instruments data acquisition board AT-MIO-16E2; Austin, TX), and stored on a hard drive. Songs were recorded and edited with LabView software (National Instruments; all custom software for this study was written by M. Rosen, F. Livingston, R. Neumann, and R. Balu). Edited songs included either one or two motifs (a motif is the largest repeated unit in the bird's song and usually comprises single and multinote syllables; zebra finch song bouts typically consist of several motifs). Stimuli presented always included the BOS and reverse BOS (i.e., song played backward) and were 1-3 sec in duration. Forward and reverse versions of the BOS contain the same spectral energy but differ in both their local and global temporal organization. Differential responsiveness to forward over reverse BOS can be used to measure neuronal sensitivity to time-varying auditory cues (see Data analysis below).

Preparatory surgery. Two days before electrophysiological recording, birds were food and water deprived for $1 \mathrm{hr}$, anesthetized with equithesin $(2 \mathrm{mg} / \mathrm{kg}$, i.m.: $0.85 \mathrm{gm}$ of chloral hydrate, $0.21 \mathrm{gm}$ of pentobarbitol, 0.42 $\mathrm{gm}$ of $\mathrm{MgSO}_{4}, 2.2 \mathrm{ml}$ of $100 \%$ ethanol, and $8.6 \mathrm{ml}$ of propylene glycol brought to a $20 \mathrm{ml}$ final volume with $\mathrm{dH}_{2} \mathrm{O}$ ), and placed in a stereotaxic device $\left(45^{\circ}\right.$ head angle; H. Adams, California Institute of Technology) The scalp was dissected along the midline, and HVc's location was determined using stereotaxic coordinates (approximately $-0.2 \mathrm{~mm}$ caudal and $2.4 \mathrm{~mm}$ lateral, measured from the caudal edge of the bifurcation of the midsagittal sinus). A stainless steel post was mounted to the rostral part of the bird's skull with dental cement, the wound was closed with cyanoacrylate, antibiotic (Neosporin ointment) was applied, and the bird was warmed under a heat lamp $\left(33^{\circ} \mathrm{C}\right)$ until recovery $(2-4 \mathrm{hr})$.

In vivo electrophysiology and song presentation. On the morning of the day of the electrophysiological recording, birds were injected in the pectoral muscle with $20 \%$ urethane $(75-105 \mu l$ total; Sigma, St. Louis, MO), administered in $25-35 \mu \mathrm{l}$ doses at $30 \mathrm{~min}$ intervals. Birds were immobilized via the mounted post in a sound-attenuating chamber (Industrial Acoustics) on an air table (Technical Manufacturing Corporation, Peabody, MA); body temperature was maintained via an electric blanket at $37^{\circ} \mathrm{C}$ (Harvard Apparatus, Holliston, MA). After topical application of xylocaine $(2 \%)$, the scalp was retracted, a small craniotomy $(<300 \mu \mathrm{m}$ wide) was made over $\mathrm{HVc}$, and the dura was slit open with a fine insect pin (Minuten, Carolina Biological Supply).

Sharp electrodes (borosilicate glass, BF100-50-10; Sutter Instrument, Novato, CA) were pulled to yield a resistance of $100-250 \mathrm{M} \Omega$ when filled with 3 M K-acetate and 5\% neurobiotin. A hydraulic microdrive (Soma Scientific, Irvine, CA) was used to lower electrodes into the nucleus $(\sim 300$ $\mu \mathrm{m}$ in depth). Brief $(\sim 1 \mathrm{msec})$ capacitance overcompensation was used to "ring" the electrode to achieve entry into the cell. An AxoClamp 2B intracellular amplifier (Axon Instruments, Foster City, CA) was used in bridge mode to record intracellular potentials, which were low-pass filtered at $3 \mathrm{kHz}$, digitized at $10 \mathrm{kHz}$, and stored on a personal computer hard drive. HVc neurons were identified on-line by their firing properties in response to injected currents (see Results) and usually by spontaneous, intermittent, high-frequency bursts of action potentials and were verified histologically after the recording session. Cells were tested with auditory stimuli if their resting potentials were negative of $-50 \mathrm{mV}$. Ten to thirty iterations of each auditory stimulus, delivered at intervals ranging from 6 to $10 \mathrm{sec}$, were presented at $\sim 70 \mathrm{~dB}$ (rms; A-weighting) through a speaker positioned $20 \mathrm{~cm}$ directly in front of the bird. Peristimulus time histograms (PSTHs; $25 \mathrm{msec}$ bin width) and median-filtered averaged membrane potential traces were computed on-line (see Data analysis) to aid in experimental decisions.

When possible, after characterization of a cell's auditory responses to BOS playback, its intrinsic properties were studied. Hyperpolarizing responses to negative current pulses $(-200$ to $-400 \mathrm{pA})$ were collected to estimate input resistance, and instantaneous and mean firing rates, as well as the latency to the first spike, were calculated in response to positive current pulses (+200 to $1000 \mathrm{pA}$; 1 sec duration) using custom LabView software. Three- to five-minute-long spontaneous membrane potential records were also collected, and a software event detector was used to estimate spontaneous firing rates. Action potential widths were measured at the base or shoulder of the spike, where the membrane potential described a sharp positive inflection. The amplitudes of spike after hyperpolarizations were measured from the spike shoulder to the trough of the hyperpolarization after the spike. Resting membrane potential was determined by subtracting any DC offset observed after electrode withdrawal from the membrane potential recorded during the 5-10 min before the end of the recording. All values are reported as the mean \pm SEM; statistics and tests for statistical significance (other than those described in Data analysis) are reported in the figure legends, Table 1, and/or the Results.

Data analysis. The suprathreshold responsiveness $\left(R_{\text {supra }}\right)$ of cells with spiking activity was calculated by $R_{\text {supra }}=S_{\mathrm{FR}}-B_{\mathrm{FR}}$, where $S_{\mathrm{FR}}$ and $B_{\mathrm{FR}}$ are the firing rates during each stimulus presentation and during a $1.5 \mathrm{sec}$ baseline period before each stimulus presentation, respectively. To assess subthreshold responsiveness in spiking and nonspiking cells, raw traces first were median-filtered (each point was replaced by the median value of the surrounding 50 points, equivalent to $5 \mathrm{msec}$ at the $10 \mathrm{kHz}$ sampling rate used here). Median-filtering removed the action potential (which was typically $\sim 1 \mathrm{msec}$ in duration) and yet did not distort slower membrane potential movements [for an example, see Jagadeesh et al. (1997), their Fig. 1]. The subthreshold depolarizing responsiveness $\left(R_{\mathrm{V}_{m}}\right)$ of these cells was measured by $R_{\mathrm{V}_{m}}=S_{\text {area }}-B_{\text {area }}$, where $S_{\text {area }}$ and $B_{\text {area }}$ are the integrals of the positive-going deviations in membrane potential either during (i.e., $S_{\text {area }}$ ) or before (i.e., $B_{\text {area }}$ ) stimulus presentation relative to the mode membrane potential measured during the baseline period. That is, the total positive area during the baseline (measured from the mode) was subtracted from the total positive area during the stimulus (measured from the mode; see Fig. 9 for examples of how these subthreshold values were measured). The mode membrane potential was calculated for the baseline data array with an automated LabView routine and was used instead of the mean or median of the baseline data array because we observed that it gave the most reliable measure of the central tendency of the baseline membrane potential. Similar calculations were made for the subthreshold hyperpolarizing area, using the negative-going deviations in membrane potential from the baseline mode value. The net subthreshold hyperpolarization was multiplied by -1 , so that a net increase in subthreshold hyperpolarization relative to the baseline would be represented by a negative number. It was possible for single cells to respond to a stimulus with an increase in both negative and positive area relative to baseline. Furthermore, strong hyperpolarizing responses to a stimulus could yield negative values for positive and negative area (e.g., when the baseline positive area was greater than the positive area evoked by the 
$A_{1} \quad x$-projecting
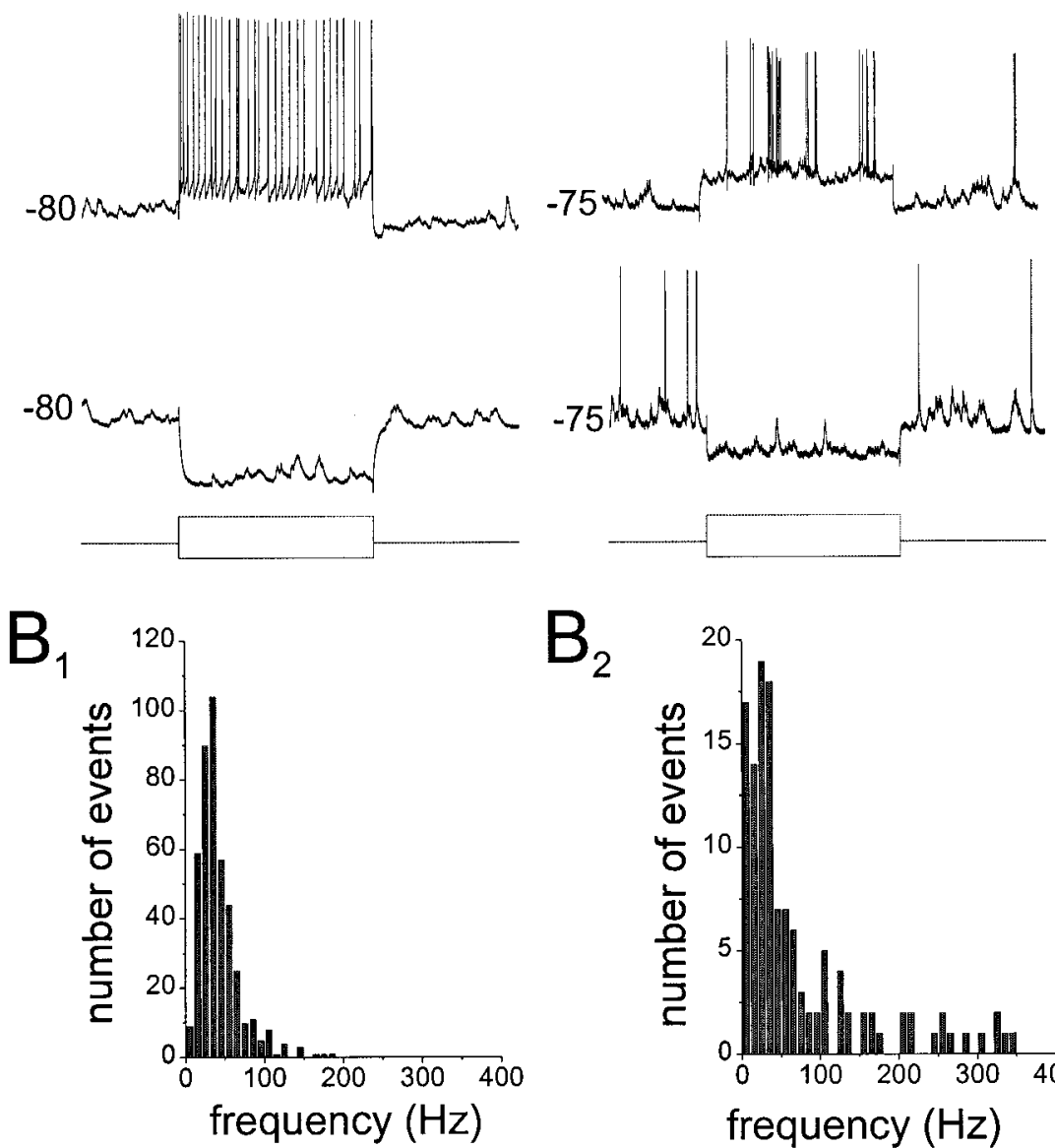

C
$A_{2}$

RA-projecting
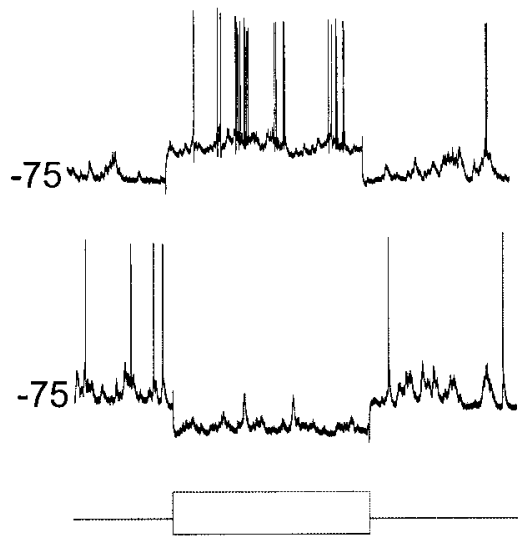

$\mathrm{B}_{2}$

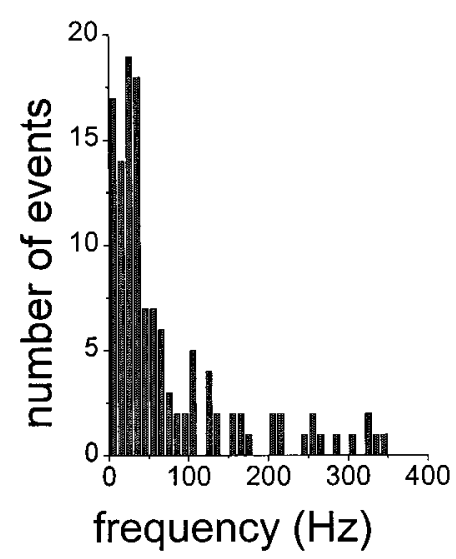

$A_{3}$

Interneuron
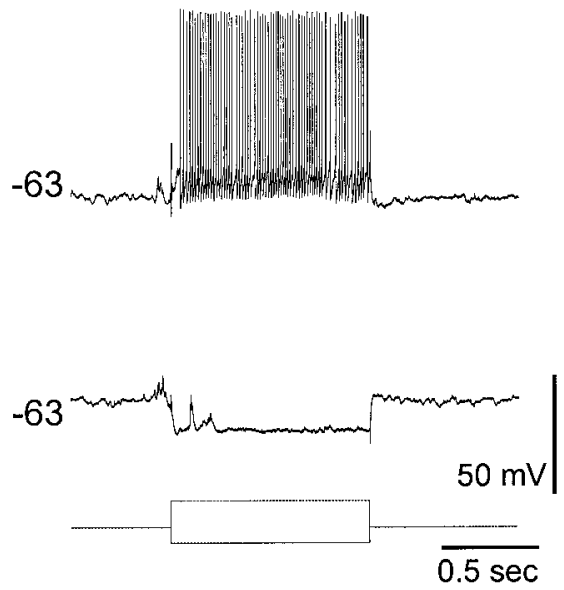

$\mathrm{B}_{3}$

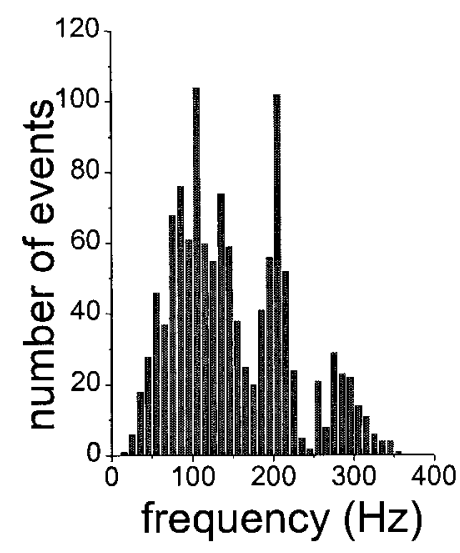

$\mathrm{B}_{4}$

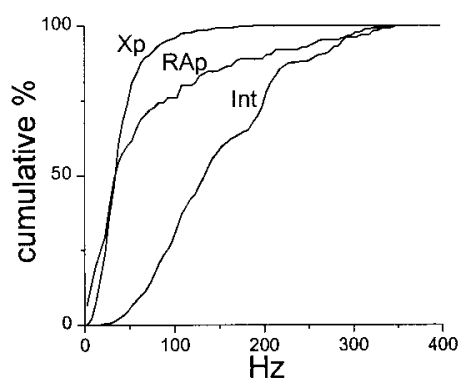

X-projecting
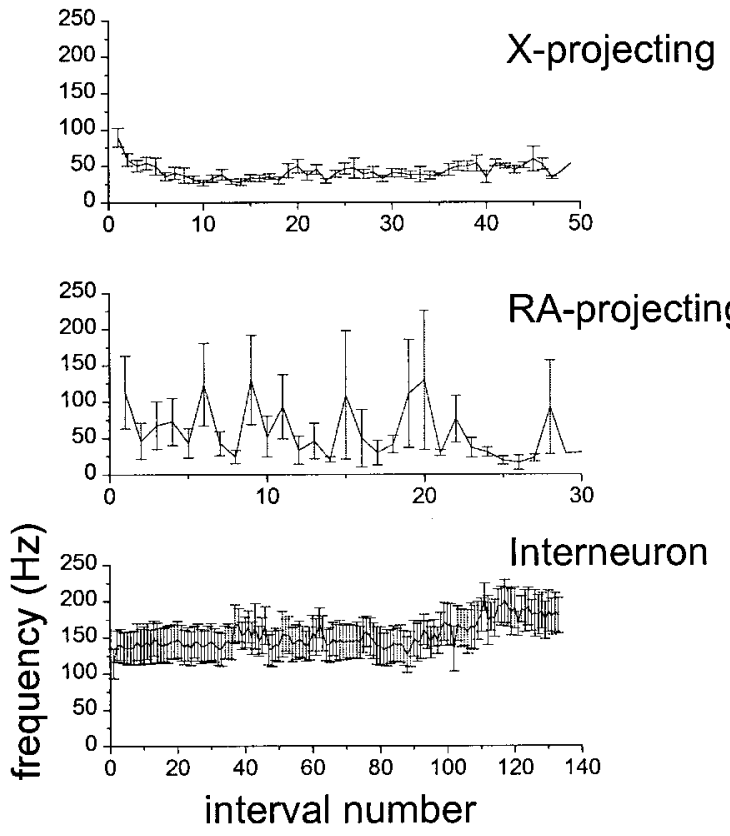

D

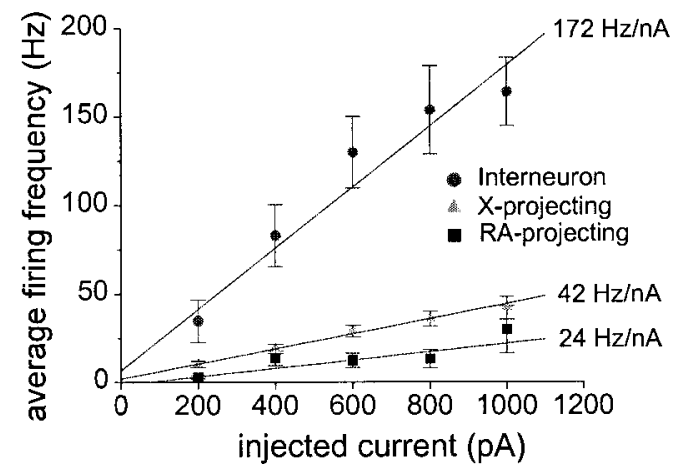

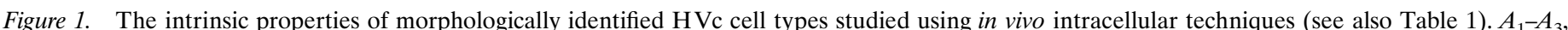

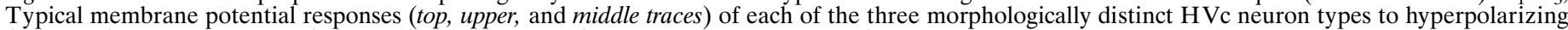

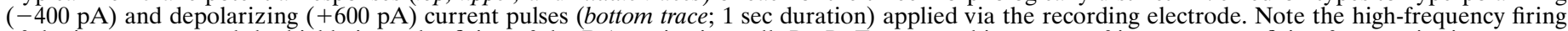

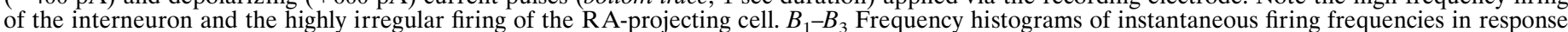

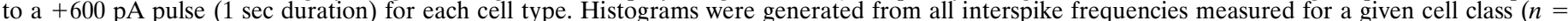

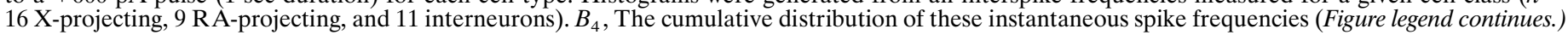


stimulus). Average $R_{\text {supra }}$ or $R_{\mathrm{V}_{m}}$ were computed for 10-30 stimulus iterations. Significance was determined with paired $t$ tests comparing stimulusevoked suprathreshold, subthreshold depolarizing or subthreshold hyperpolarizing responses to corresponding baseline measures.

To compare suprathreshold and subthreshold responses, response strengths were expressed as z-scores. The suprathreshold z-score $\left(Z_{\text {supra }}\right)$ is given by the difference between the average firing rate during stimulus presentation and that during a $1.5 \mathrm{sec}$ baseline period before stimulus presentation, divided by the SD of this difference:

$$
Z_{\text {supra }}=\frac{\bar{S}_{\mathrm{FR}}-\bar{B}_{\mathrm{FR}}}{\sqrt{\operatorname{Var}\left(S_{\mathrm{FR}}\right)+\operatorname{Var}\left(B_{\mathrm{FR}}\right)-2 \operatorname{Covar}\left(S_{\mathrm{FR}}, B_{\mathrm{FR}}\right)}}
$$

where $\bar{S}_{\mathrm{FR}}$ is the mean firing rate during the stimulus, $\bar{B}_{\mathrm{FR}}$ is the mean firing rate during the baseline period, and the denominator is the SD of $S_{\mathrm{FR}}-B_{\mathrm{FR}}$. For nonspiking cells and median-filtered spiking cells, the subthreshold z-score $\left(Z_{V_{V}}\right)$ is given by the difference between the average area during stimulus presentation and that during baseline, divided by the $\mathrm{SD}$ of this difference. The $Z_{\mathrm{V}_{m}}$ formula is the same as that for $Z_{\text {supra }}$ with substitutions of ${ }_{\text {area }}$ for ${ }_{\mathrm{FR}}$, where $\bar{S}_{\text {area }}$ is the mean deviation in $\mathrm{V}_{m}$ (from the baseline mode, calculated separately for negative or positive area, as stated above) during song presentation and $\bar{B}_{\text {area }}$ is the mean deviation in $\mathrm{V}_{m}$ during baseline; the denominator is the SD of $S_{\text {area }}-B$

The selectivity of a given neuron for forward over reverse BOS playback was measured using the psychophysical metric $d^{\prime}$ (Green and Swets, 1966), which estimates the discriminability between two stimuli. A difference in response to these two stimuli has been used previously as the criterion for the auditory selectivity of neurons in $\mathrm{HVc}$, as well as in other song nuclei (Solis and Doupe, 1997; Theunissen and Doupe, 1998; Janata and Margoliash, 1999; Rosen and Mooney, 2000). The $d^{\prime}$ value comparing the response to BOS relative to reverse BOS is given by:

$$
d_{\text {supra }}^{\prime}=\frac{2\left(\bar{R}_{\mathrm{FR} \text { воs }}-\bar{R}_{\mathrm{FR} \mathrm{rev}}\right)}{\sqrt{\sigma^{2} \mathrm{BOS}+\sigma^{2} \mathrm{rev}}} \text { or } d_{\mathrm{Vm}}^{\prime}=\frac{2\left(\bar{R}_{\text {areaвos }}-\bar{R}_{\text {arearev }}\right)}{\sqrt{\sigma^{2} \mathrm{BOS}+\sigma^{2} \mathrm{rev}}}
$$

where $d^{\prime}{ }_{\text {supra }}$ represents suprathreshold responsiveness and $d^{\prime}{ }^{\prime}{ }_{m}$ represents subthreshold responsiveness. $\bar{R}$ is the mean value of $R$ (as described above), and $\sigma^{2}$ is its variance. This measure of selectivity is similar to a ratio measure but takes into account both the mean and the variance of a cell's responses and can report negative values. A $d^{\prime}$ value $>0.7$ (or $<$ -0.7 , reflecting a relative excitatory bias toward reverse BOS) was used as the criterion for identifying a cell as "selective"; this corresponded to a significance level of $p=0.036$ as measured by a paired $t$ test comparing $R_{\mathrm{V}_{m}}$ or $R_{\mathrm{FR}}$ values for 20 presentations of BOS versus reverse BOS. Note that $d^{\prime}$ values for subthreshold responses $\left(d^{\prime}{ }_{\mathrm{V}}\right)$ were calculated separately for positive and negative areas. Tests for statistical significance are reported in the figure legends, except where noted in the Results.

To quantify the relative spike timing of the different cell types during BOS playback, the action potential PSTH of an RA-projecting neuron was correlated (Origin, Microcal Corporation) with the PSTH of either an $\mathrm{X}$-projecting neuron or interneuron recorded from within the same bird. To examine the relationship between spike timing and membrane polarity (i.e., interneuron spiking vs X-projecting neuronal polarity), the crosscorrelation analysis was performed using the interneuron action potential PSTH and the median-filtered average membrane potential of an X-projecting cell from the same bird; the membrane potential trace was further divided into 25 -msec-long average "points" [i.e., the trace was divided into sequential segments 250 points in length $(25 \mathrm{msec}$ at the 10 $\mathrm{kHz}$ sample rate), and the average of these 250 points was calculated to yield a single point every $25 \mathrm{msec}$, equaling the temporal resolution of the PSTH]. In each case, the cross-correlation analysis was performed on data collected during forward BOS playback. Each cross-correlation was performed using data collected from a single-cell pair within a single bird, each resultant cross-correlogram was normalized to its peak value, and the normalized values from a given pair type (e.g., RA-projecting vs $\mathrm{X}$-projecting) were then averaged across all pairs that were obtained (i.e., across birds).

Histology. Cells were filled with neurobiotin using positive currents $(+0.5$ to $+1 \mathrm{nA} ; 500 \mathrm{msec}$ at $1 \mathrm{~Hz})$. After the recording session, birds were deeply anesthetized with equithesin and transcardially perfused with $0.9 \%$ saline for $3 \mathrm{~min}$, followed by $4 \%$ paraformaldehyde (PFA) in $25 \mathrm{~mm}$ sodium phosphate buffer for $30 \mathrm{~min}$. Brains were removed and post-fixed in $4 \%$ PFA with $30 \%$ sucrose overnight, blocked sagittally, and sectioned on a freezing microtome at $60 \mu \mathrm{m}$. Sections either were processed using a standard HRP-DAB reaction technique (see Kittelberger and Mooney, 1999) or occasionally were visualized using avidin-Oregon green (Molecular Probes, Eugene, OR; sections were incubated overnight at $4^{\circ} \mathrm{C}$ in a 1:500 dilution of avidin-Oregon green and $0.4 \%$ Triton X-100 in $0.025 \mathrm{M}$ PBS, rinsed four times for $20 \mathrm{~min}$ each in PBS, mounted, and coverslipped). Camera lucida drawings were made using both $10 \times$ and $63 \times$ (Zeiss; $1.3 \mathrm{nA}$ ) objectives. Cell bodies were traced under the $63 \times$ objective, and their diameters were measured along the major axis of the cell body using an eyepiece reticule. A neuron's dendritic extent also was estimated using the eyepiece reticule and was measured from the middle of the cell body to the tip of the longest dendrite in the same section. Only DAB-reacted cells were used for dendritic measurements; because of this, as well as some incomplete fills, only a subset of labeled cells were included for this analysis.

\section{RESULTS}

Intracellular recordings were made in the $\mathrm{HVc}$ of urethaneanesthetized adult male zebra finches to (1) establish the relationship between an HVc neuron's intrinsic electrophysiological properties and its morphological type, (2) use this information to determine which HVc neuron types are auditory, (3) examine the subthreshold events that contribute to song-selective action potential responses in different HVc neurons, and (4) enable within-bird comparisons between the song-evoked responses in different $\mathrm{HVc}$ neuron types.

\section{Intrinsic properties of morphologically identified HVc neurons}

Previous in vitro studies indicated that different morphological classes of $\mathrm{HVc}$ neurons in adult male zebra finches possess distinct intrinsic electrophysiological properties (Dutar et al., 1998). The existence of similar distinctions in vivo would enable sorting of the cells to class on the basis of their intrinsic properties alone, especially advantageous when intracellular recordings are too brief to afford good staining $(<15 \mathrm{~min}$ ) or when many cells are impaled and filled in a single nucleus, making an unambiguous assignment of an electrophysiological record to a dye-filled cell difficult. Therefore, the in vivo intrinsic properties of $\mathrm{HVc}$ neurons (morphologically identified post hoc via intracellular neurobiotin staining; see below) were first characterized by collecting spontaneous membrane potential records and by injecting them with hyperpolarizing and depolarizing currents (Fig. $1 A ; n=16 \mathrm{X}$-projecting, $10 \mathrm{RA}$ projecting, and 11 interneurons). The three morphological classes of $\mathrm{HVc}$ neurons had distinct intrinsic properties in vivo, differing in the distributions of their instantaneous spike frequencies in response to similar injected currents (Fig. $1 A-C$ ), their average firing frequencies in response to depolarizing current pulses (Fig. 1D), their spontaneous firing rates, their action potential widths, the amplitude of their individual spike afterhyperpolarizations (AHPs), and the onset latencies to their first action potential during a depolarizing pulse (Table 1). Interneurons were readily distinguished from the two relay cell types by their extremely high-frequency, nonadapting action potential trains in response to depolarizing pulses (Fig. $1 A-C$ ), their markedly steeper average firing frequencies in response to these injected currents (Fig. $1 D$, Table 1), their narrower action potential widths, their larger spike

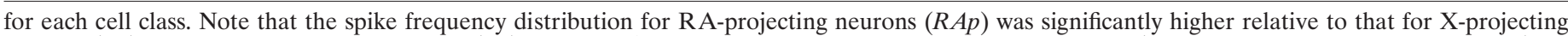

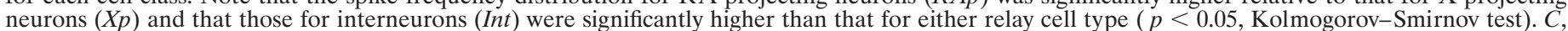

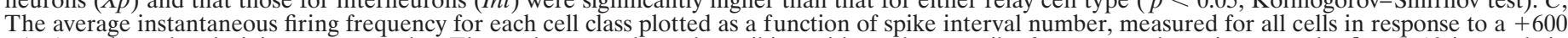

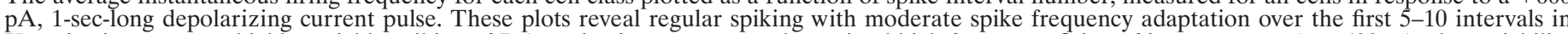

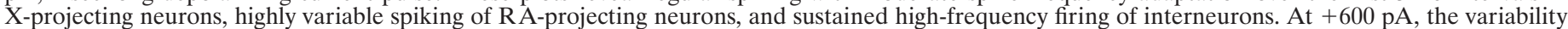

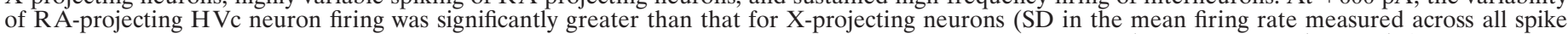

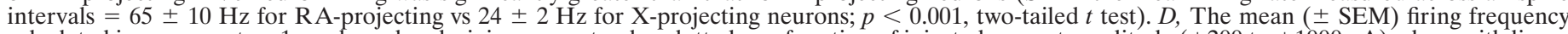

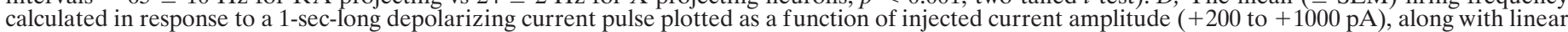

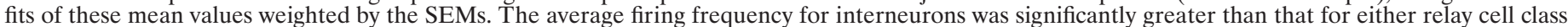

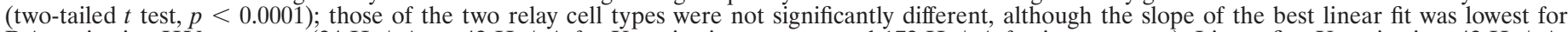

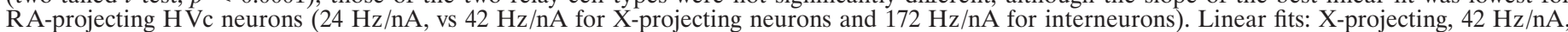

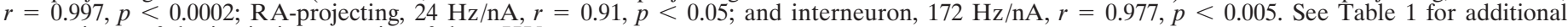
comparisons of the intrinsic properties of these HVc neuron types. 
A

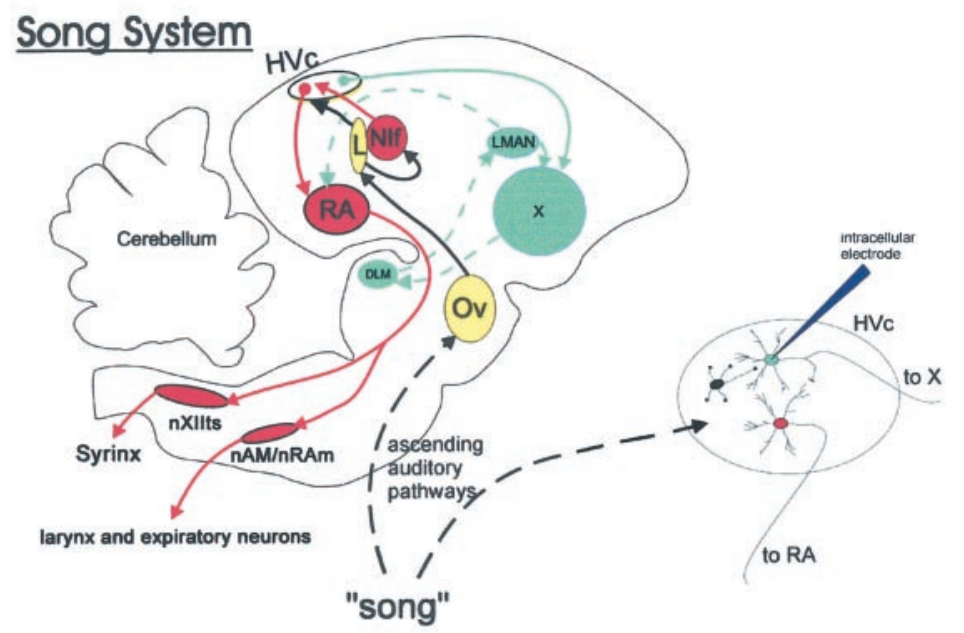

B

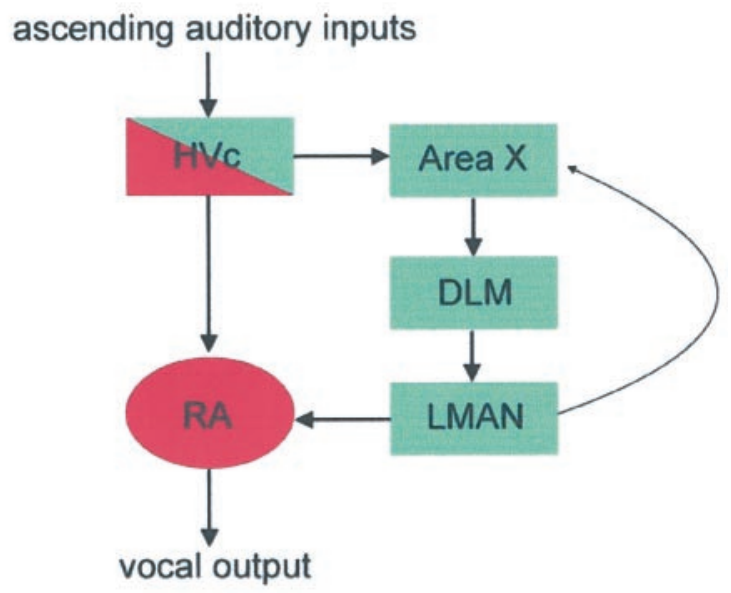

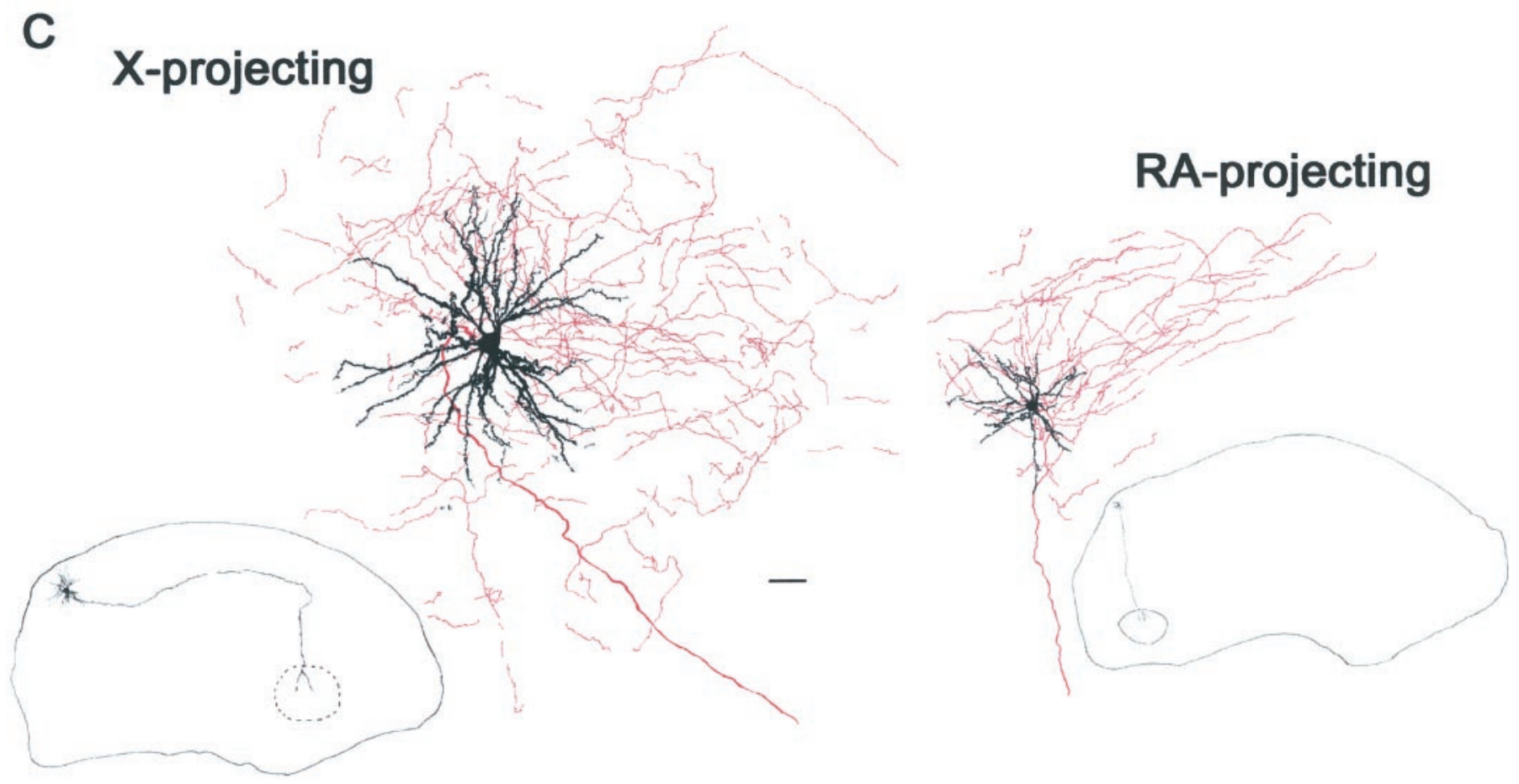

Figure 2. Schematic of the song system, the connectivity of $\mathrm{HVc}$, and the morphology of $\mathrm{HVc}$ relay cell types revealed via in vivo intracellular staining with neurobiotin. $A$, A sagittal view of the song system, as well as the ascending auditory pathway (shown in highly abbreviated form by the dashed line) that provides auditory input to HVc. HVc contains two relay cell populations: the first (shown in red) projects to the vocal premotor nucleus RA, which innervates the hypoglossal motor neurons and respiratory premotor neurons used for singing, and the second (shown in blue) projects to the basal ganglia homolog area X, giving rise to the anterior forebrain pathway, which includes the thalamic nucleus DLM and the anterior telencephalic nucleus LMAN, which in turn innervates RA. Besides the two relay cell types, HVc contains local interneurons (shown as a small, dark blue cell in the inset). Field L, the primary telencephalic thalamorecipient auditory area, is the likely source of auditory input to $\mathrm{HVc}$, either directly or indirectly via NIf, a sensorimotor structure that is a major afferent to HVc (see Janata and Margoliash, 1999), and/or via an interposed area (the "shelf") that is contiguous to the ventral border of HVc. B, A flowchart depicting the major functional divisions in the forebrain song control circuitry that arise from the two relay cell populations in $\mathrm{HVc}$, including the vocal motor pathway (red) and the anterior forebrain pathway (blue). $C$, Representative examples of the $\mathrm{HVc}$ relay cell types that served as the focus of this study. Camera lucida reconstructions of RA-projecting and X-projecting neurons that were stained with neurobiotin using in vivo intracellular techniques are shown. These reconstructions (drawn under a $63 \times$ objective, $1.3 \mathrm{nA}$ ) show the cell body and dendrites in black and the main axon and local axon collaterals in red. The low-power $(2.5 \times$ objective $)$ drawings show the path of the main axon of the given relay cell type traveling from the parent cell body to the postsynaptic target (i.e., nucleus RA or area X). Both cell types had spinous dendrites and extensive local axon collaterals. Scale bar: high power, $30 \mu \mathrm{m}$; low power, $750 \mu \mathrm{m}$; sections are in the sagittal orientation. DLM, medial nucleus of the dorsolateral thalamus; $H V c$ (used as a proper name); $L$, field $\mathrm{L} ; L M A N$, lateral magnocellular nucleus of the anterior neostriatum; $n A M$, nucleus ambiguus; NIf, nucleus interfacialis; $n R A m$, nucleus retroambigualis; $n X I I t s$, tracheosyringeal portion of the hypoglossal nucleus; $O v$, nucleus ovoidalis; $R A$, robust nucleus of the archistriatum; $X$, area X.

AHPs, and their higher rates of spontaneous firing (Table 1). $\mathrm{X}$-projecting neurons also fired relatively regular action potential trains when depolarized (Fig. $1 A$ ), but with moderate spike frequency adaptation over the first 5-10 spikes (Fig. 1C), a shallower current-to-spike-frequency relationship (Fig. $1 D$, Table 1), broader action potentials, and lower rates of spontaneous firing (Table 1). Finally, unlike X-projecting neurons, RA-projecting neurons exhibited highly variable (Fig. $1 A, C$ ) spike timing during depolariz- 


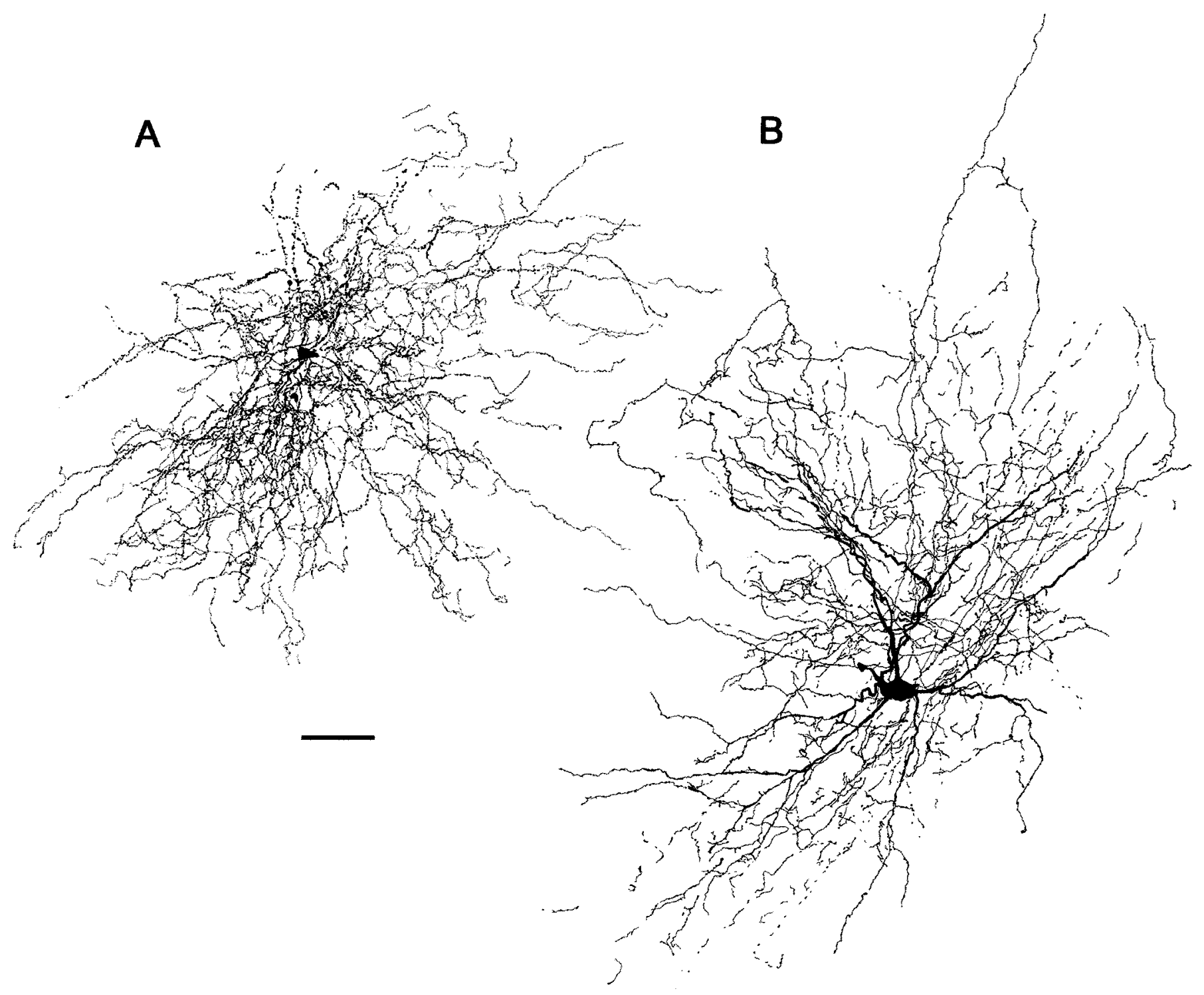

Figure 3. A camera lucida reconstruction of the two types of HVc interneurons studied here. A, The most common interneuron encountered here had varicose processes, without a distinct axon, and a small cell body (10/11 cells). $B$, This cell is an HVc interneuron with a morphology distinct from that of the more common type; it had smooth rather than beaded dendrites and fine axon collaterals that were restricted to HVc. Both were fast-spiking cells and had otherwise similar electrophysiological properties (see Results). Scale bar, $30 \mu \mathrm{m}$.

ing current injection; these evoked responses could include very high-frequency bursts $(>200 \mathrm{~Hz}$ : Fig. $1 A, B)$. In further contrast to both X-projecting neurons and interneurons, RA-projecting $\mathrm{HVc}$ neurons displayed firing behavior that could be described as "sluggish," as reflected in their significantly longer and more variable latencies to first spike onset when injected with positive current (Table 1) and the trends these cells manifested toward both the lowest average current-to-frequency relationship (Fig. 1D, Table 1) and the lowest mean rates of spontaneous firing (Table 1). In practice, this sluggish behavior along with the highly irregular action potential discharge of RA-projecting neurons in response to moderate positive currents (approximately $+500 \mathrm{pA}$ ) was the most useful feature for distinguishing them from $\mathrm{X}$-projecting neurons on-line, whereas the fast-spiking behavior of interneurons enabled them to be readily distinguished from the two relay cell classes. Input resistances, as well as resting potentials, were not significantly different for the three cell classes (Table 1).

\section{Morphology}

In vivo intracellular neurobiotin staining revealed that the two $\mathrm{HVc}$ relay cell types differed morphologically from one another, as well as from interneurons (see Figs. 2-4). Differences among all three cell types included the presence or absence of a projection axon, the projection axon's termination, cell body size, dendritic extent, and the relative abundance or absence of dendritic spines. These differences are very similar to those that have been noted in previous morphological analyses of HVc neurons (Nixdorf et al., 1989; Fortune and Margoliash, 1995; Benton et al., 1998).

The main axons of X- and RA-projecting HVc neurons had different trajectories and termini, projecting either rostrally from $\mathrm{HVc}$ to area X or caudally to nucleus RA, whereas interneurons elaborated processes wholly restricted to HVc (see Figs. 2, 3). The main axons of almost all (14/16) X-projecting neurons traveled immediately in the rostral direction after exiting HVc (Fig. 2C). These rostral trajectories were quite varied, including dorsal paths that initially ran along the ventricle, above the lamina hyperstriatica $(\mathrm{LH})$, as well as more ventral routes within and between the $\mathrm{LH}$ and the lamina medullaris dorsalis. X-projecting axons that described the most dorsal trajectories turned sharply in the ventral direction close to the rostrocaudal plane of the lateral magnocellular nucleus of the anterior neostriatum (LMAN) and then traveled near or through LMAN en route to area X (an example of this is shown in Fig. $2 C$ ). In two cases, X-projecting axons traveled 


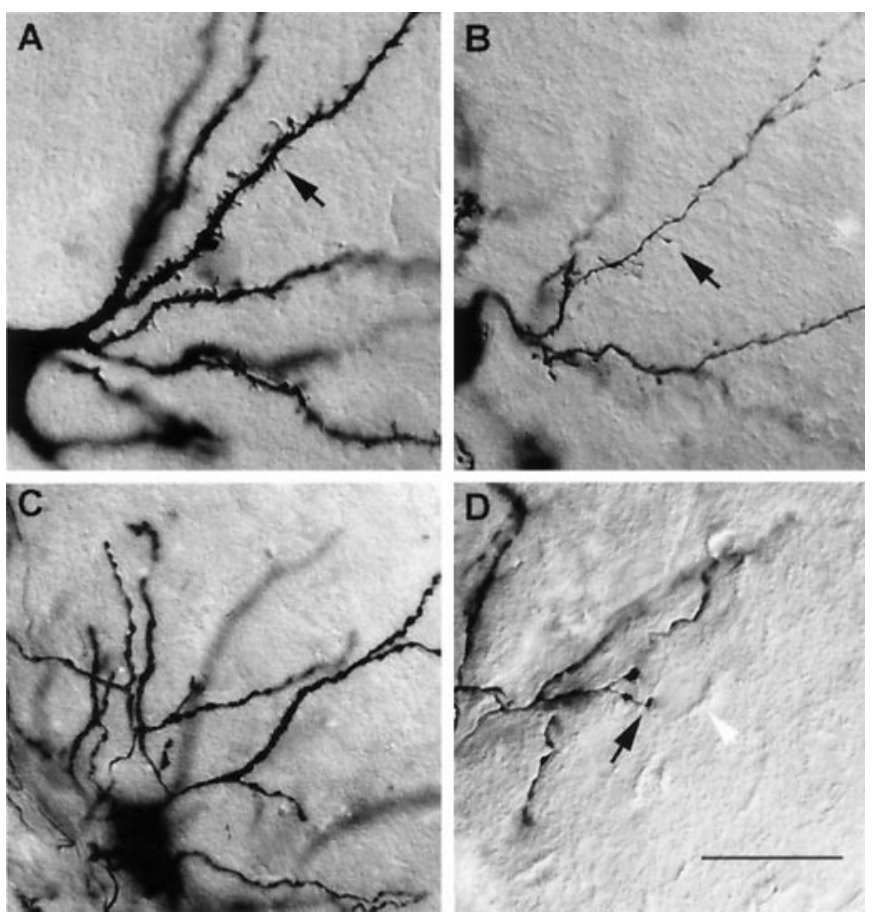

Figure 4. Photomicrographs illustrate the morphological differences between the different types of $\mathrm{HVc}$ relay cells and interneurons, as seen under high-magnification $(100 \times, 1.4 \mathrm{nA}$ objective) differential interference contrast optics. $A$, The dendrites of X-projecting $\mathrm{HVc}$ neurons were thick and heavily spined (arrow). B, The dendrites of RA-projecting $\mathrm{HVc}$ neurons were thinner and more sparsely spined, and individual spines could be long-necked (arrow). C, The most common type of HVc interneuron encountered here had swellings along the length of individual processes. $D$, A putative axosomatic contact between a terminal swelling (black arrow) of a process extending within $\mathrm{HVc}$ from the more common interneuron type and an unstained cell body (white arrowhead) is shown. Scale bars: $A, B, D$, $30 \mu \mathrm{m} ; C, 45 \mu \mathrm{m}$.

ventrally into the archistriatum after leaving $\mathrm{HVc}$ and then ran rostrally along the ventral floor of the telencephalon toward area X. Both of these ventral-going axons followed a more medial trajectory in their descent from HVc and were medial to RA at the point they initiated their rostral turn toward area X. In all RA-projecting neurons $(10 / 10$ cells $)$, the main axon traveled ballistically in a caudal and ventral direction via the tractus dorsalis archistriatalis to RA (Fig. 2C). Both relay cell types elaborated extensive axon collaterals within $\mathrm{HVc}$, indicating that they are likely to function as local circuit neurons in $\mathrm{HVc}$ as well as serving as relay cells to other song nuclei (Fig. 2C). In contrast to the two relay cell types, $\mathrm{HVc}$ interneurons had processes entirely restricted to $\mathrm{HVc}$; usually, an interneuron's dendrites and axons were difficult to distinguish from one another (Figs. 3, 4).

In agreement with previous studies indicating that X-projecting neurons have larger cell bodies than other $\mathrm{HVc}$ neuron types (Paton et al., 1985; Alvarez-Buylla et al., 1988), the cell body diameters of X-projecting neurons stained here were larger than those of interneurons and RA-projecting neurons (soma diameters: $\mathrm{X}$-projecting, $23.0 \pm 0.9 \mu \mathrm{m} ; n=16$ cells; interneurons, $18.7 \pm 1.0$ $\mu \mathrm{m} ; n=10$; RA-projecting, $16.8 \pm 0.8 \mu \mathrm{m} ; n=9$; Xp $>$ Int, $p<$ 0.005; Xp $>$ RAp, $p<0.0001$; unpaired $t$ test). Qualitatively, the cell bodies of RA-projecting neurons had more rounded profiles than had those of both X-projecting neurons and interneurons, which tended to be polyhedral or fusiform in shape. Both relay cell types had spinous dendrites, but the dendrites of RA-projecting neurons were more compact (Fig. 2; mean of greatest radial dendritic extent: X-projecting, $168 \pm 4 \mu \mathrm{m} ; n=12$ cells; RAprojecting, $123 \pm 0.9 \mu \mathrm{m} ; n=6$ cells; $p<0.001$, unpaired $t$ test) and were thinner and more sparsely spined (Figs. 2, 4). The RA-projecting neurons stained here strongly resemble the short dendrite class of $\mathrm{HVc}$ neurons described in the canary $\mathrm{HVc}$, which were tentatively classified from Golgi-impregnated material as RAprojecting on the basis of their small soma sizes (Nixdorf et al., 1989). In contrast to the two relay cell types, all $11 \mathrm{HVc}$ interneurons had aspinous processes. The processes of 10 of the 11 interneurons had swellings, or varicosities, along their lengths (Figs. $3 A$, $4 C$ ). Swellings on some interneuronal processes could be seen in close apposition to other cell bodies, suggestive of axosomatic synapses (Fig. 4D). The one remaining interneuron had smooth dendrites, which were readily distinguished from a thinner axon and its very fine collaterals (Fig. $3 B$ ). This cell had the highest average firing-frequency-to-current relationship of any interneuron measured $(\sim 390 \mathrm{~Hz} / \mathrm{nA})$, although its action potential width, AHP height, input resistance, and resting potential fell within the range described for the other 10 interneurons. The combination of fastspiking electrophysiological properties and varicose, aspinous dendrites seen in $\mathrm{HVc}$ interneurons is reminiscent of inhibitory interneurons that have been described in other song areas (Spiro et al., 1999) and in the mammalian telencephalon (Azouz et al., 1997; Thomson and Deuchard, 1997).

\section{Auditory responses of identified HVc neurons}

In vivo intracellular recordings were made from a total of 172 neurons situated within and around HVc. Many of these cells were held too briefly to characterize their auditory responses or to identify the cell type. Sixty neurons were sorted unambiguously to type relying on both morphological and electrophysiological criteria, and their auditory responses were characterized using forward and reverse playback of the BOS. These identified HVc neurons included $20 \mathrm{X}$-projecting neurons (all confirmed morphologically), 23 RA-projecting neurons (11 confirmed morphologically), and 17 interneurons ( 7 confirmed morphologically).

Previous extracellular studies have shown that the BOS is a potent auditory stimulus for many HVc neurons (Sutter and Margoliash, 1994; Volman, 1996). Here, all identified HVc neuron types were found to respond robustly to forward playback of the BOS, firing significantly above the baseline rate during presentation of the stimulus (firing rate during BOS $>$ baseline firing rate, $p<0.05,17 / 20 \mathrm{X}$-projecting neurons; 15/23 RA-projecting neurons; $14 / 17$ interneurons). Representative responses to BOS playback are shown for each cell type in Figure 5, which includes individual raw current-clamp records collected during five consecutive playbacks of the BOS. These individual traces were used to construct both the action potential PSTHs as well as the medianfiltered, averaged membrane potential records to quantify a neuron's subthreshold and suprathreshold responses to song playback (see Materials and Methods).

Several features distinguished the song-evoked auditory responses of the three HVc cell types (Fig. 5). First, the majority of BOS-evoked action potential responses in both relay cell classes were phasic, occurring at restricted points during a motif (see Figs. 5, 6, 8A, B, top; 10/17 X-projecting neurons and 9/15 RA-projecting neurons that fired significantly to forward BOS did so in a phasic manner). In contrast, almost all interneurons exhibited BOS playback-evoked action potential responses that were sustained throughout the stimulus, or throughout each motif (see Figs. 5, 6; $12 / 14$ interneurons that responded significantly to forward BOS fired in a sustained manner to the stimulus). Second, all RAprojecting neurons encountered here showed robust subthreshold responses to BOS playback (i.e., the BOS-evoked depolarizing response $>$ spontaneous depolarizations before playback; $p<0.05$; $23 / 23$ cells). Even the eight RA-projecting neurons that did not show elevated firing to BOS playback (including three cells that evinced neither spontaneous nor stimulus-evoked firing) still exhibited significant subthreshold depolarizing responses to BOS. Third, the subthreshold responses of RA-projecting neurons were always strongly depolarizing from rest $(23 / 23$ cells $)$, in contrast to those of X-projecting cells, which could include hyperpolarizing (7/20 cells; $p<0.05$ ) or depolarizing ( $8 / 20$ cells; $p<0.05$ ) components (see Materials and Methods and below). These recordings demonstrate that all three $\mathrm{HVc}$ cell types have auditory responses 

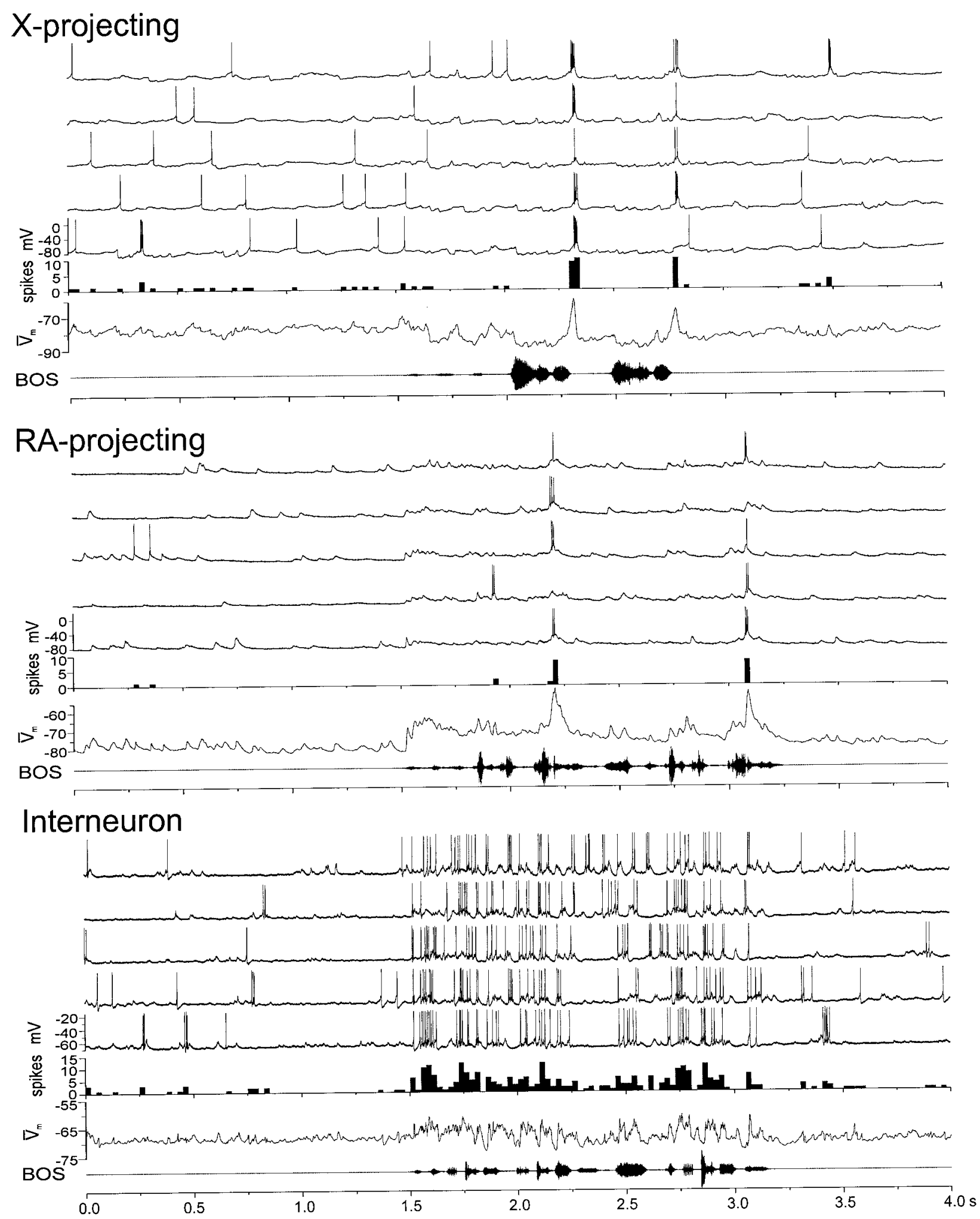

Figure 5. Representative responses of identified $\mathrm{HVc}$ neurons to auditory presentation (playback) of the BOS. For each cell type (X-projecting, RA-projecting, and interneuron), the top five traces in each panel are the individual current-clamp records collected during five consecutive BOS playbacks (shown as an oscillogram at the bottom of each panel and preceded by a $1.5 \mathrm{sec}$ silent period). Immediately below the current-clamp records are the PSTHs (spikes) of spiking activity and the median-filtered, average membrane potential generated from the raw traces ( $\overline{\mathrm{V}}_{\mathrm{m}}$; see Materials and Methods). For both the X-projecting and RA-projecting neurons shown here, song-evoked action potential responses were highly phasic, consisting of short bursts of action potentials, whereas the accompanying subthreshold changes in membrane potential were distinctly different; spiking in the X-projecting neuron was preceded by membrane hyperpolarization below the resting membrane potential, whereas spiking in the RA-projecting neuron was preceded by depolarization above its resting membrane potential. In contrast to the phasic responses of the two relay cells, the interneuron displayed more sustained action potential discharge throughout BOS playback (spike height in this example was truncated because of capacitive filtering, which more greatly attenuated action potential height in these fast-spiking cells than in relay cells). All of these cells were morphologically identified after the recording session using intracellular neurobiotin staining and were collected from three different animals. 


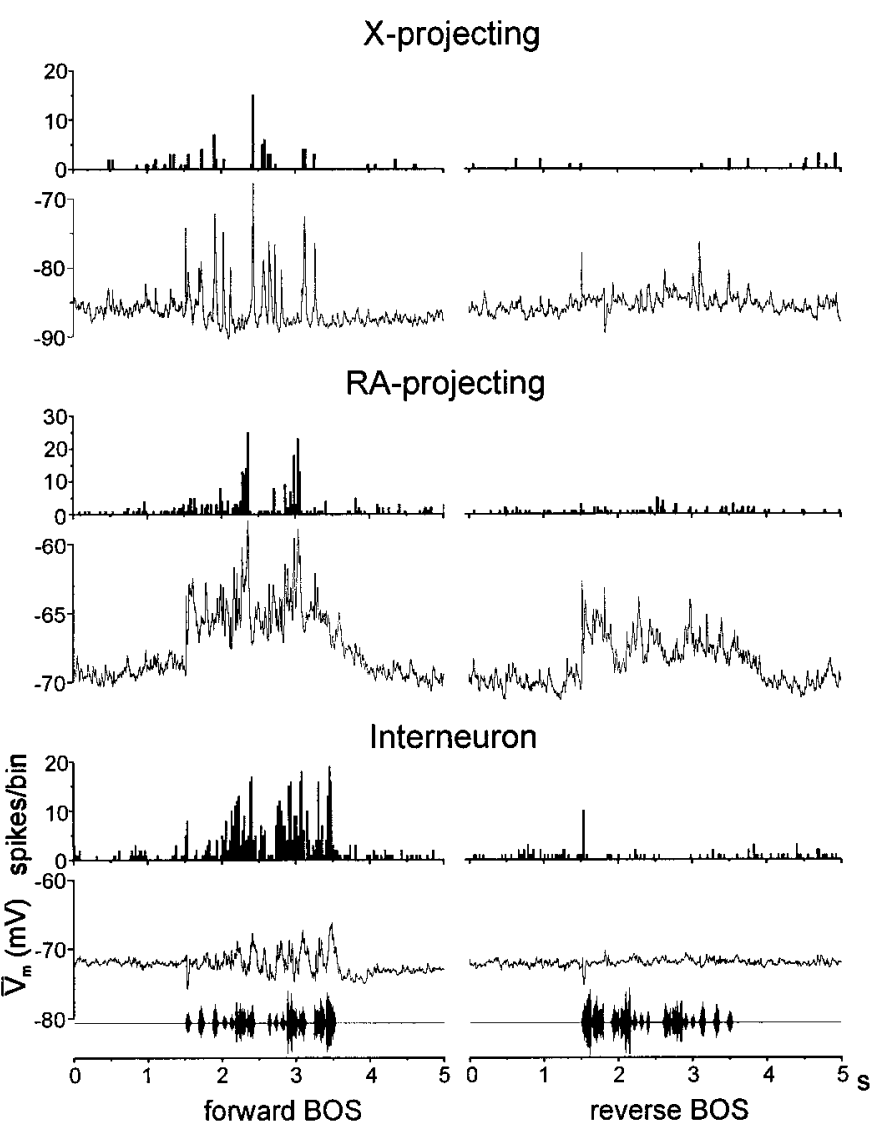

Figure 6. In HVc, X-projecting and RA-projecting neurons as well as interneurons are song selective, displaying enhanced action potential firing to forward over reverse BOS playback. For each cell type, the PSTHs (spikes/bin) and median-filtered averaged membrane potential traces $\left[\overline{\mathrm{V}}_{\mathrm{m}}\right.$ $(\mathrm{mV})]$ are shown. These three cells were collected from a single bird; responses are to 20 iterations each of the forward and reverse BOS playback, shown as oscillograms at the bottom of the figure. For the $\mathrm{X}$-projecting neuron, slight membrane hyperpolarization and highly phasic subthreshold depolarizations were evoked by forward BOS playback, with only minor subthreshold depolarizations accompanying reverse BOS playback. For the RA-projecting neuron, both forward and reverse BOS playback elicited subthreshold depolarizations, although only forward BOS evoked a significantly elevated action potential response. For the interneuron, only forward BOS playback elicited subthreshold or suprathreshold responses. The X-projecting neuron and interneuron were identified morphologically, whereas the RA-projecting cell was identified on the basis of its intrinsic electrophysiological properties.

and raise the possibility that all three types generate BOS-evoked firing via different synaptic mechanisms.

\section{Song selectivity}

Birdsongs vary in their temporal as well as spectral organization. Previous extracellular studies have shown that many $\mathrm{HVc}$ neurons are sensitive to time-varying acoustical features of the bird's own song, firing selectively for forward over reverse BOS playback (these two stimuli contain the same spectral energy but differ in their temporal organization) (Margoliash, 1986; Lewicki and Arthur, 1996; Volman, 1996; Theunissen and Doupe, 1998). Here, intracellular recordings confirmed that all three HVc neuron types could fire selectively for forward over reverse BOS playback [Fig. 6; $d^{\prime}>0.7,18 / 20$ X-projecting neurons; 15/23 RA-projecting neurons; $15 / 17$ interneurons (one interneuron that did not display significant firing-rate elevation to forward BOS did display significant firing-rate suppression to reverse BOS)].

The median-filtered average membrane potential traces in the three cells shown in Figure 6 illustrate that distinct subthreshold membrane potential responses accompanied their song-selective firing. For example, the interneuron and the X-projecting cell underwent depolarizing and hyperpolarizing movements to for- ward song, with little subthreshold responsiveness to reverse BOS playback. In contrast, the RA-projecting neuron displayed substantial subthreshold depolarizations to reverse as well as forward BOS playback.

\section{Relationships between subthreshold and suprathreshold selectivity}

As a first step toward identifying differences in the subthreshold auditory responsiveness of the three $\mathrm{HVc}$ neuron types, their subthreshold depolarizations and firing-rate responses to forward and reverse BOS playback were measured. Z-scores were calculated for depolarizing (positive) subthreshold areas (see Materials and Methods) and firing-rate responses to forward and reverse BOS for individual neurons of each HVc neuron class. For individual cells, either subthreshold area or firing-rate $\mathrm{z}$-scores for forward BOS responses were plotted against those same measures of reverse BOS responses. These comparisons revealed that subthreshold versus suprathreshold responsiveness varied systematically according to $\mathrm{HVc}$ cell type (Fig. 7A). Both X-projecting neurons and interneurons exhibited firing-rate responses biased to forward BOS, without a similar bias in their depolarizing subthreshold responses. In contrast, RA-projecting neurons displayed firing-rate and depolarizing subthreshold responses biased to forward over reverse BOS playback (see Fig. 7 and legend for statistical tests of significance).

The different patterns of subthreshold and suprathreshold responsiveness of the three $\mathrm{HVc}$ neuron types to forward and reverse BOS playback suggest that X-projecting neurons and interneurons produce more selective action potential output than would be predicted from their depolarizing subthreshold responses alone. To confirm that this was indeed the case, subthreshold and firing-rate $d^{\prime}$ statistics (see Materials and Methods) were computed to allow direct comparisons of subthreshold versus firing-rate selectivity within single $\mathrm{HVc}$ neurons of a given class. Plotting $d^{\prime}$ values for depolarizing subthreshold responses (area) against those for firing rate revealed that selectivity was greater at a firing-rate level than at a subthreshold level for X-projecting neurons and interneurons but lower at a firing-rate than subthreshold level for RA-projecting neurons (Fig. 7B; see figure and legend for relevant statistics). At a population level, mean $d^{\prime}$ values for firing rate in the three $\mathrm{HVc}$ cell types greatly exceeded 0.7 (i.e., were selective for forward over reverse BOS playback), with the two relay cell classes displaying almost identical absolute levels of firing-rate selectivity (mean $d^{\prime}$, $\mathrm{X}$-projecting, $1.35 \pm 0.26$; RA-projecting, $1.34 \pm 0.17$; interneuron, $3.05 \pm 0.44)$. In almost every case, selectivity stemmed from an augmentation of firing rate in response to forward BOS either alone or in combination with firing-rate suppression to reverse BOS, rather than only a suppression of firing rate in response to reverse BOS (selective cells with an absolute excitatory bias to forward BOS, 18/18 X-projecting neurons; 15/15 RA-projecting neurons; 14/15 interneurons). In contrast, significantly elevated firing-rate responses to reverse $\mathrm{BOS}$ playback were rare in all song-selective $\mathrm{HVc}$ neurons ( $p<0.05$; $0 / 18 \mathrm{X}$-projecting neurons; 1/15 RA-projecting neurons; 3/14 interneurons).

\section{Membrane potential effects on BOS-evoked subthreshold and action potential responses}

BOS playback could elicit hyperpolarizing responses in some X-projecting neurons (Figs. 5, 6) (see also Lewicki, 1996), while evoking exclusively depolarizing responses in RA-projecting neurons (Figs. 5, 6). To test whether different patterns of song-evoked inhibition and excitation distinguish song-selective responses in the two H Vc relay cell types, tonic-positive current was injected via the recording electrode to depolarize the resting membrane potential of individual HVc neurons during song playback (Fig. 8). These membrane potential manipulations were used to unmask or augment potentially latent song-evoked inhibition and to determine whether subthreshold depolarizations that occurred at the cell's normal resting potential were excitatory or reversed inhibitory events. BOS-evoked excitation and inhibition were assessed by 

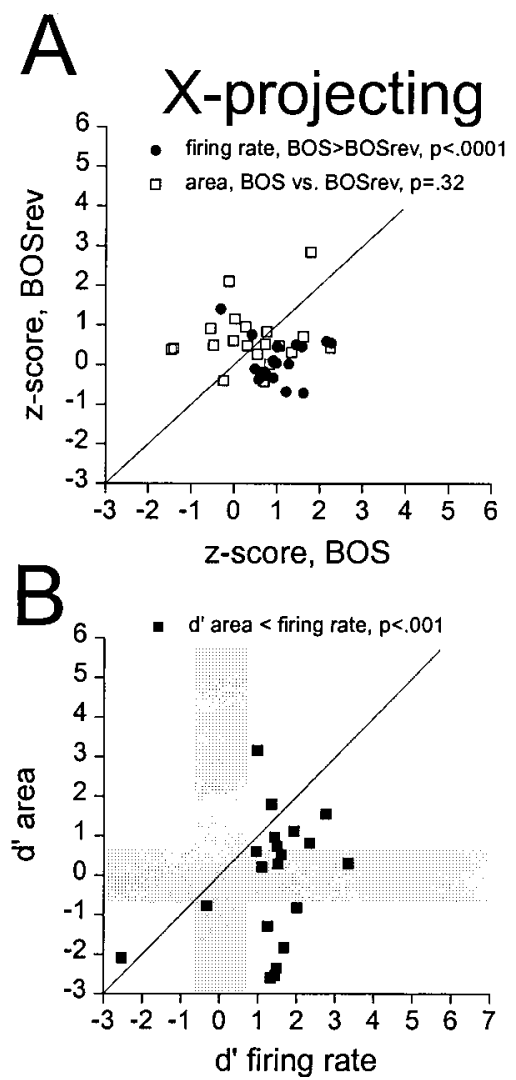

RA-projecting
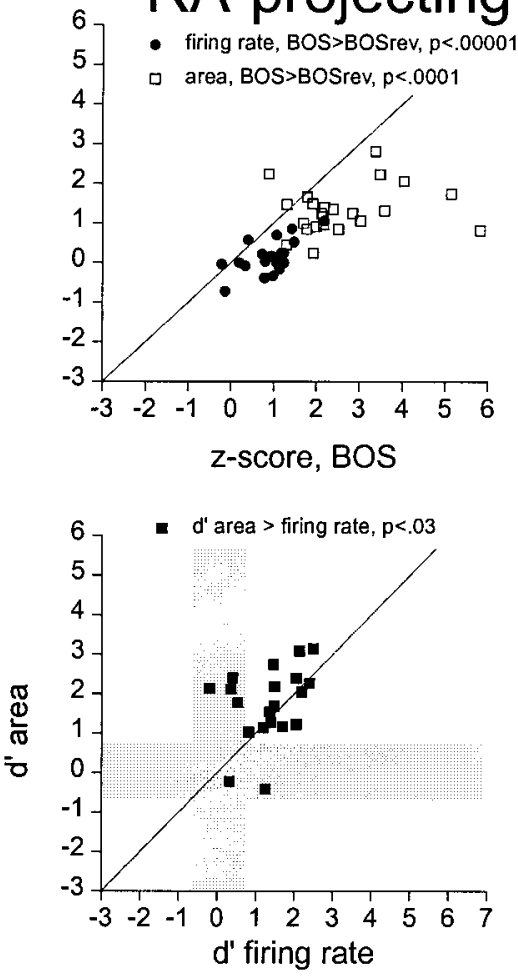

\section{Interneuron}
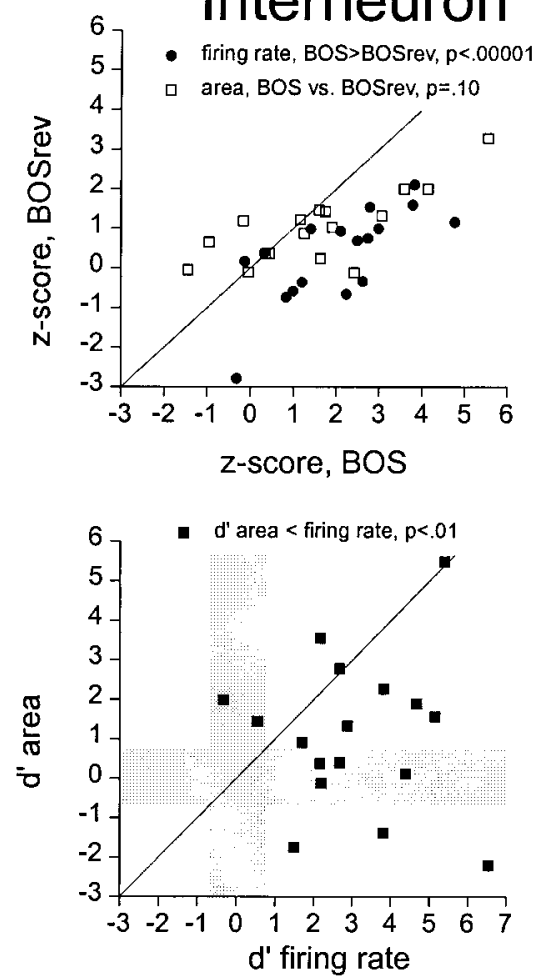

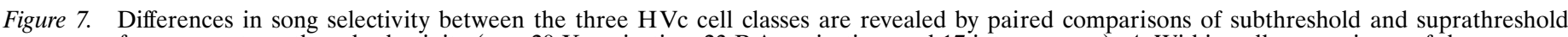

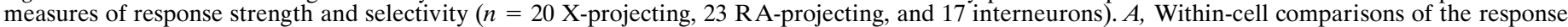

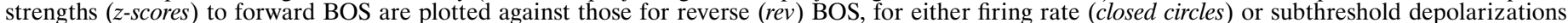

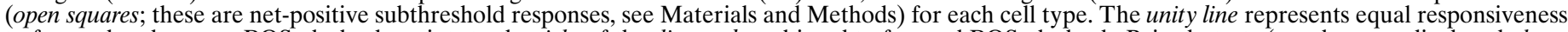

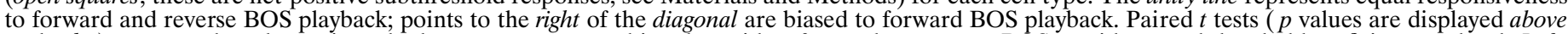

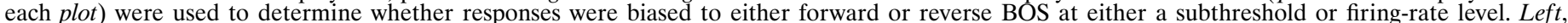

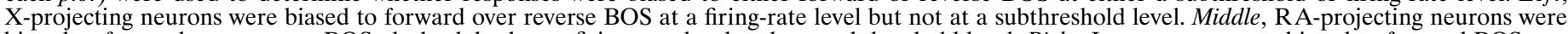

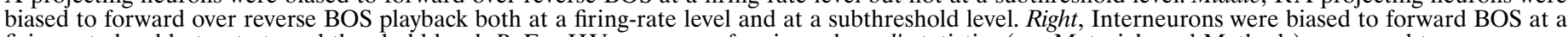

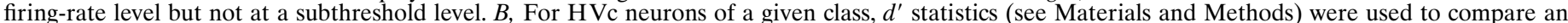

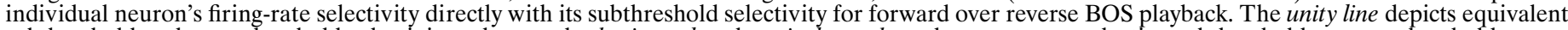

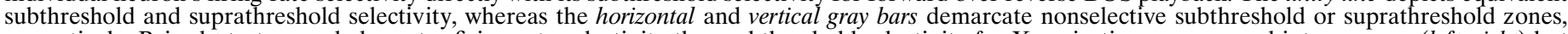

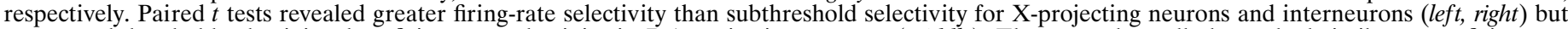

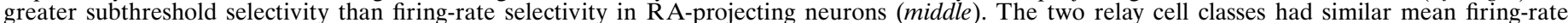

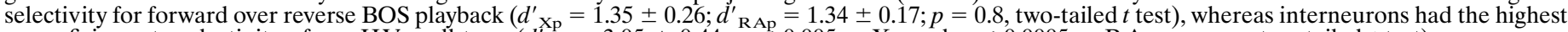
mean firing-rate selectivity of any HVc cell type $\left(d_{\text {Int }}^{\prime}=3.05 \pm 0.44 ; p<0.005\right.$ vs Xp and $p<0.0005$ vs RAp neurons, two-tailed $t$ test $)$.

measuring both subthreshold and firing-rate responses for the two types of relay cells at their normal resting potentials and when the cells were tonically depolarized. To measure subthreshold depolarizations and hyperpolarizations, positive and negative areas between the median-filtered average membrane potential traces and the prestimulus baseline mode potential were estimated before and during playback, and the positive or negative prestimulus area was then subtracted from the stimulus-evoked area of the same sign to yield net subthreshold responses (see Materials and Methods; Fig. 9). In parallel to these subthreshold measurements, firing rates to forward and reverse BOS playback relative to baseline rates were calculated at the two different membrane potentials. Manipulating membrane potential in this way revealed different patterns of subthreshold activity accompanied by firing-rate suppression or augmentation in the two cell types (Figs. 8, 9).

Tonic depolarization of $\mathrm{X}$-projecting neurons resulted in periods of pronounced membrane hyperpolarization to forward BOS playback that were paralleled by action potential suppression, suggestive of an inhibitory synaptic mechanism (Fig. 8A). For $\mathrm{X}$-projecting neurons at their normal resting potentials, forward BOS playback could evoke either net depolarizing (4/11 cells; $p<$ $0.05)$ or net hyperpolarizing $(3 / 11$ cells; $p<0.05)$ subthreshold responses relative to baseline fluctuations in membrane potential (Fig. $9 A)$. Tonically depolarizing these cells $\left(\mathrm{V}_{\text {normal }}=-76.5 \pm 2.1\right.$
$\mathrm{mV}$ vs $\mathrm{V}_{\text {depolarized }}=-57.6 \pm 3.5 \mathrm{mV} ; p<0.001 ; n=11$ cells) strongly augmented BOS-evoked hyperpolarization, such that all 11 cells displayed only net hyperpolarizing responses to forward BOS playback (Fig. $9 A$; net hyperpolarizing, $p<0.05$; 11/11 cells; net depolarizing, $p<0.05$; 0/11 cells; see Fig. 9 legend for mean z-scores for negative and positive areas). Although highly phasic depolarizing responses to forward BOS could still persist during tonic-positive current injection (Fig. $8 A ; \sim 2.7 \mathrm{sec}$ ), they failed to exceed spontaneous baseline depolarizations in total area. Subthreshold responses of any kind to reverse BOS were rare, and tonic depolarization did not unmask latent inhibition (or excitation) evoked by this stimulus (Fig. $8 A$; population data not shown; net depolarizing, $p<0.05 ; 2 / 11$ cells at normal $\mathrm{V}_{m}$ and $1 / 11$ cells at positive $\mathrm{V}_{m}$; net hyperpolarizing, $p<0.05 ; 2 / 11$ cells at normal $\mathrm{V}_{m}$ and $1 / 11$ cells at positive $\mathrm{V}_{m}$ ).

In X-projecting neurons, the subthreshold effects of altering membrane potential were paralleled by changes in BOS-evoked firing (Figs. $8 A, 9 B$ ). At normal resting membrane potentials, significant firing-rate increases above baseline accompanied forward BOS playback in all 11 cells, whereas only one of these cells displayed a significant suprathreshold response to reverse BOS, which consisted of firing-rate suppression. During tonic depolarization, forward BOS playback evoked either firing-rate suppression (BOS-evoked firing rate $<$ baseline firing rate; $p<0.05 ; 5 / 11$ 
A

X-projecting: control

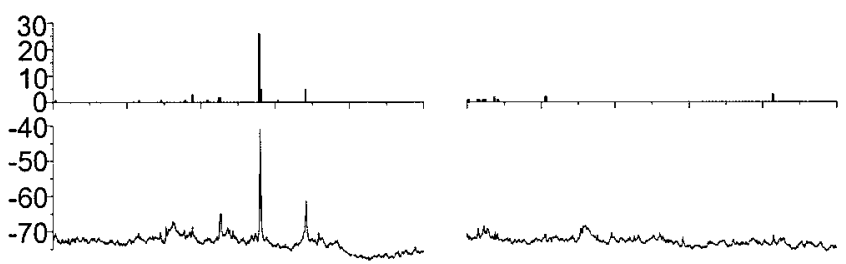

$X$-projecting: depolarized

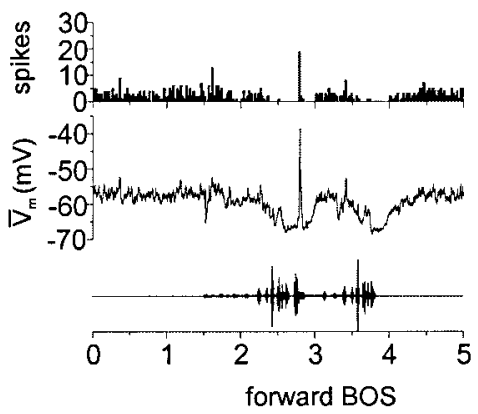

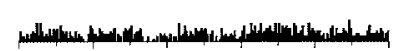
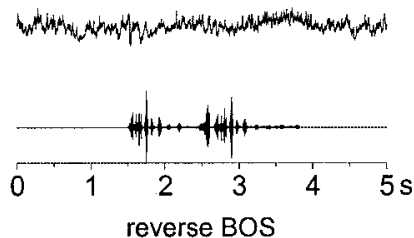

B

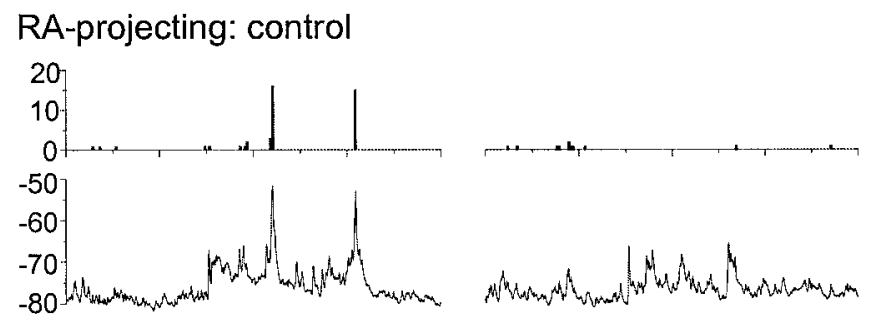

RA-projecting: depolarized

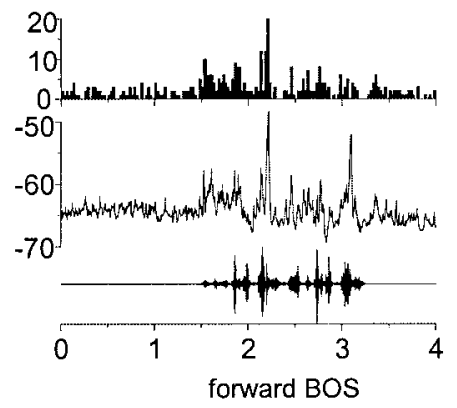

Figure 8. Tonic depolarization unmasked latent inhibition evoked by forward BOS playback in an X-projecting neuron and latent excitation to forward and reverse BOS playback in an RA-projecting neuron. The PSTHs and median-filtered average membrane potential traces were generated from current-clamp records obtained during 20 iterations of forward and reverse BOS playback (oscillograms at bottom of each panel), presented either at the cell's unmanipulated resting membrane potential (control) or with tonic depolarizing current applied via the recording electrode to positively shift the cell's membrane potential (depolarized). A, For an X-projecting neuron at its normal resting potential, forward BOS playback evoked a highly phasic action potential response that was paralleled by a similarly phasic subthreshold depolarization. In the tonically depolarized state, forward BOS playback evoked firing-rate suppression paralleled by membrane hyperpolarization, suggestive of a previously latent inhibitory mechanism. Reverse BOS playback did not evoke subthreshold or suprathreshold responses to playback at either the control or depolarized membrane potentials. $B$, For an RA-projecting neuron observed at its unmanipulated resting potential, highly phasic action potential responses occurred only in response to forward BOS playback, but pronounced and sustained subthreshold depolarizations were evoked by both forward and reverse BOS. Tonic depolarization resulted in elevated action potential discharge to both forward and reverse BOS playback, indicating that the subthreshold depolarizations seen at the normal resting potential were latent excitatory events. Note that the subthreshold traces in the depolarized state do not reveal the BOS-evoked hyperpolarization seen in the X-projecting neuron. Population data for these effects are shown below (see Fig. 9).

cells) or no significant suprathreshold response of any kind relative to baseline firing rates ( $p>0.05 ; 6 / 11$ cells), whereas none of these cells displayed significant firing-rate responses to reverse BOS in the tonically depolarized state $(p>0.5 ; 11 / 11$ cells $)$. Paired comparisons of $\mathrm{z}$-scores for firing rate in response to either forward or reverse BOS playback at the two membrane potentials show that tonic depolarization selectively altered action potential responses to forward, but not reverse, BOS (Fig. 9B). Forward BOS-evoked firing-rate responses changed from net positive (i.e., augmentation) to net negative (i.e., suppression) relative to baseline with depolarization, whereas reverse BOS did not evoke significant suprathreshold responses in either the resting or depolarized state (mean $\mathrm{Z}$-scores, $\mathrm{Z}_{\text {normalBos }}=0.972 \pm 0.103$ vs $\mathrm{Z}_{\text {depolarizedBos }}=-0.731 \pm$ 0.336; $p<0.001$, paired $t$ test; $Z_{\text {normalBosrev }}=-0.090 \pm 0.118$ vs $\mathrm{Z}_{\text {depolarizedBoSrev }}=0.121 \pm 0.186 ; p=0.24$, paired $t$ test; $n=11$ cells). Taken together, the subthreshold and suprathreshold effects of changing membrane potential are consistent with the idea that $\mathrm{X}$-projecting neurons receive excitatory and inhibitory inputs activated primarily by forward BOS playback but do not receive inputs of either sign that are strongly activated by reverse BOS playback.

Unlike X-projecting neurons, membrane potential manipulations in RA-projecting neurons suggest that they receive predominantly excitatory inputs strongly activated by both forward and reverse BOS playback. At their normal resting membrane potentials, RAprojecting neurons exhibited exclusively depolarizing subthreshold responses to forward as well as reverse BOS playback (Figs. 8B, 9A; net depolarizing, $p<0.05$; forward BOS, $8 / 8$ cells; reverse BOS, $8 / 8$ cells; net hyperpolarizing, $p<0.05$; forward BOS, $0 / 8$ cells; reverse BOS, 0/8 cells). Unlike X-projecting neurons, tonic depolarization $\left(\mathrm{V}_{\text {normal }}=-79.6 \pm 2.8 \mathrm{mV}\right.$ vs $\mathrm{V}_{\text {depolarized }}=-57.4 \pm 3.2 \mathrm{mV} ; p<$
$0.0001 ; n=8$ cells) did not reveal robust forward BOS-evoked inhibition (Figs. $8 B, 9 A$; net depolarizing, $p<0.05$; forward BOS, 5/8 cells; reverse BOS, 4/8 cells; net hyperpolarizing, $p<0.05$; forward BOS, $2 / 8$ cells; reverse BOS, $0 / 8$ cells). Of the two RAprojecting neurons that did display song-evoked hyperpolarization, one simultaneously maintained a net positive subthreshold response, while the other exhibited the highest stimulus-evoked firing rate observed for any RA-projecting neuron, inconsistent with an inhibitory input. One possibility is that, at higher firing frequencies, spike AHPs contributed significantly to the median-filtered traces and increased the average membrane potential negativity.

$\mathrm{RA}$-projecting neurons differed from X-projecting neurons in that tonic depolarization almost always augmented their songevoked firing, especially to reverse BOS, and never resulted in firing-rate suppression to forward BOS playback (Figs. 8B, 9B). Six of seven RA-projecting neurons displayed significant firing-rate responses to forward BOS playback at their normal resting membrane potentials, and five of these cells exhibited higher absolute firing rates to forward BOS in the depolarized state. In addition, a seventh cell that did not display a significant firing-rate response to forward BOS playback at either membrane potential still exhibited a $>15$-fold increase in response strength after depolarization $\left(\mathrm{Z}_{\text {normal }}=0.33 ; p=0.33\right.$; vs $\left.\mathrm{Z}_{\text {depolarized }}=5.38 ; p=0.07\right)$. As a group, the mean firing-rate response of RA-projecting neurons to forward BOS playback more than doubled after depolarization, although this effect fell short of significance (mean $\mathrm{Z}$-scores, $\mathrm{Z}_{\mathrm{con}}$ trolBos $=1.01 \pm 0.15 ; Z_{\text {depolarizedBos }}=2.34 \pm 0.60 ; p=0.12$, paired $t$ test; $n=6$; two cells did not spike in the depolarized state. In RA-projecting neurons, firing-rate suppression was never observed in response to forward BOS playback, regardless of mem- 


\section{A) Vm effects on subthreshold responses}
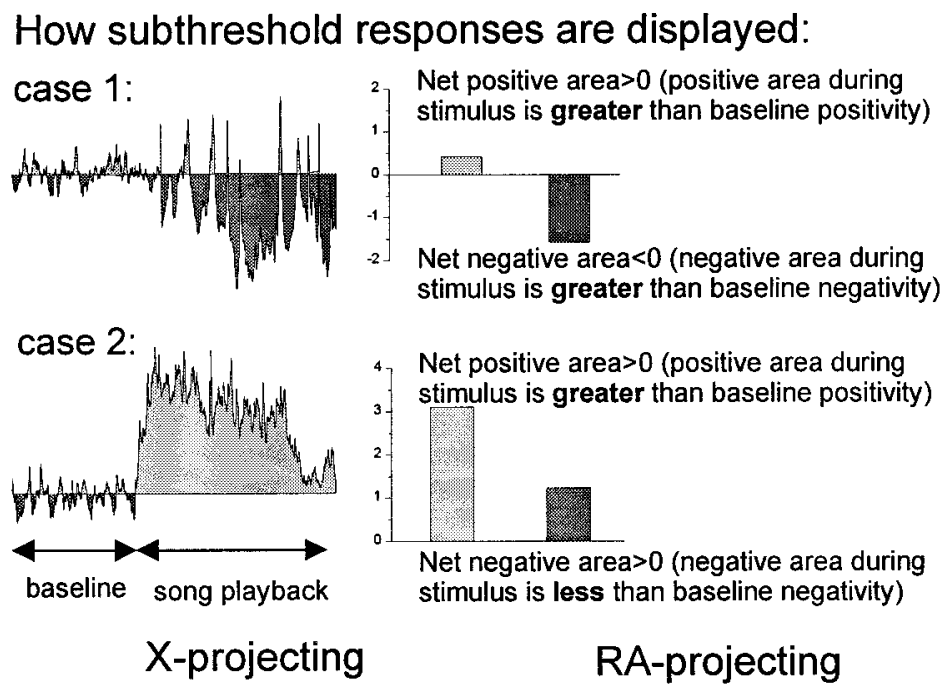

Net negative area $>0$ (negative area during stimulus is less than baseline negativity)
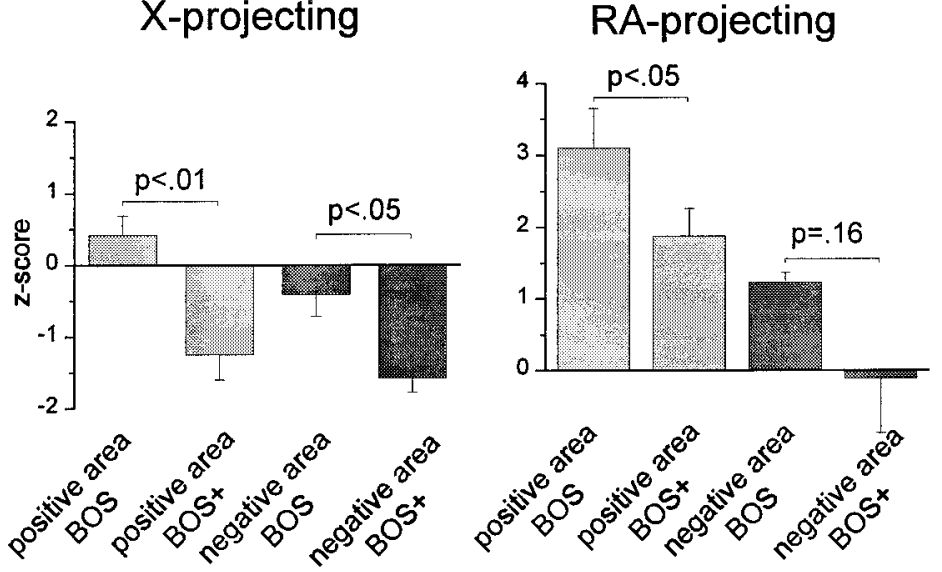

\section{B) Effects of Vm on firing rate}
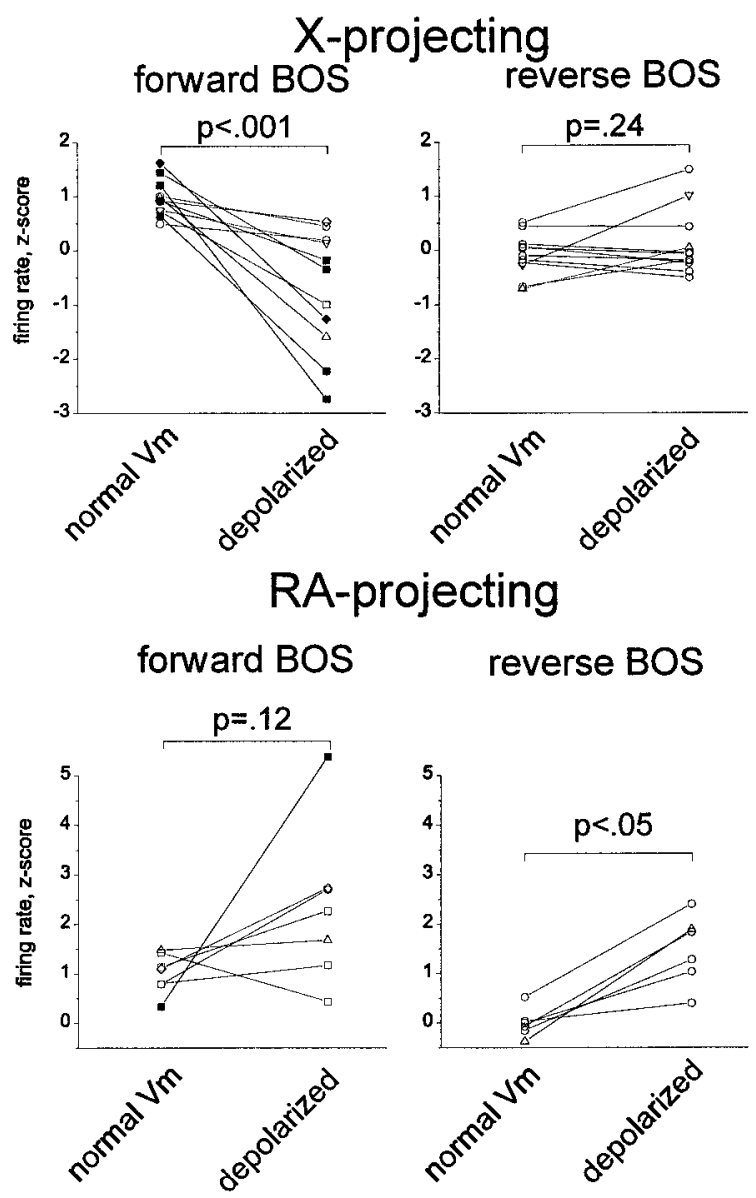

reverse BOS

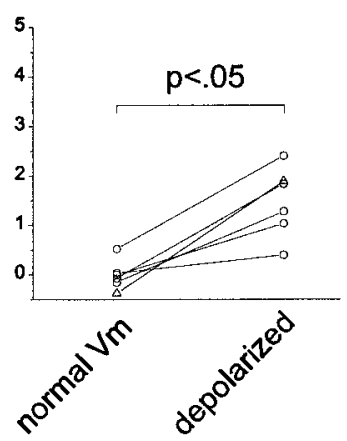

Figure 9. Population analyses of the effects of tonic depolarization on subthreshold responses in the two relay cell types indicate that X-projecting neurons display a mixture of inhibition and excitation evoked primarily by forward BOS, whereas RA-projecting neurons display predominantly excitatory responses to both forward and reverse BOS. A, Top, An example of how the positive and negative subthreshold responses to forward and reverse BOS playback were measured is shown. Positive or negative z-scores were generated from positive or negative integrals calculated relative to a baseline mode value, either before or during stimulus presentation; the prestimulus positive area was then subtracted from the BOS-evoked positive area to generate a positive area score (lightly shaded; values $>0$ correspond to a net increase in positivity during stimulation). A similar subtraction was performed with the negative integrals, which were then multiplied by -1 to generate a negative area score (darkly shaded; values $<0$ correspond to a net increase in negativity during playback). Bottom Left, The means $( \pm$ SEM) of the subthreshold positive and negative area Z-scores for X-projecting neurons in their control and tonically depolarized states $\left(\mathrm{V}_{\text {control }}=-76.5 \pm 2.1 \mathrm{mV}\right.$ vs $\mathrm{V}_{\text {depolarized }}=-57.6 \pm 3.5 \mathrm{mV} ; p<0.001 ; n=11$ cells $)$ are shown. Depolarization altered the responses of $\mathrm{X}$-projecting neurons so that they were net negative. Positive area: $\mathrm{Z}_{\text {areaBoscontrol }}=0.42 \pm 0.27 ; \mathrm{Z}_{\text {areaBosdepolarized }}=-1.25 \pm 0.35 ; p<0.01$, paired $t$ test. Negative areas: $Z_{\text {areaBoscontrol }}=-0.41 \pm 0.30 ; Z_{\text {areabosdepolarized }}=-1.58 \pm 0.20 ; p<0.05$, paired $t$ test. Bottom Right, Subthreshold positive and negative area $\mathrm{Z}$-scores $( \pm \mathrm{SEM})$ for RA-projecting neurons in their control and tonically depolarized states $\left(\mathrm{V}_{\text {control }}=-79.6 \pm 2.8 \mathrm{mV}\right.$ vs $\mathrm{V}_{\text {depolarized }}=-57.4 \pm 3.2 \mathrm{mV} ; p<0.0001 ; n=8$ cells) are shown. Mean responses were highly positive in either state. Positive areas: $Z_{\text {areaBoscontrol }}=$ $3.1 \pm 0.55 ; \mathrm{Z}_{\text {areaBosdepolarized }}=1.87 \pm 0.38 ; p<0.05$, paired $t$ test. Negative areas: $\mathrm{Z}_{\text {areaBoscontrol }}=1.23 \pm 0.13 ; \mathrm{Z}_{\text {areaBosdepolarized }}=-0.11 \pm 0.76 ; p=$ 0.16 , paired $t$ test. $B$, Top, In X-projecting neurons, tonic depolarization significantly depressed firing rates to forward BOS playback, without affecting firing-rate responses to reverse BOS playback. Mean firing rates: $\mathrm{Z}_{\text {controlBos }}=0.97 \pm 0.10$ versus $\mathrm{Z}_{\mathrm{depolarizedBos}}=-0.73 \pm 0.34 ; p<0.001$, paired $t$ test; $\mathrm{Z}_{\text {controlBoSrev }}=-0.09 \pm 0.12$ versus $Z_{\text {depolarizedBoSrev }}=0.12 \pm 0.19 ; p=0.24 ; n=11$ cells. Bottom, In RA-projecting neurons, tonic depolarization resulted in a significantly elevated firing-rate response to reverse BOS playback and a trend toward elevated firing to forward BOS playback. Mean firing rates: $\mathrm{Z}_{\text {controlBos }}=1.01 \pm 0.15 ; \mathrm{Z}_{\text {depolarizedBos }}=2.34 \pm 0.60 ; p=0.12$, paired $t$ test; $\mathrm{Z}_{\text {controlBoSrev }}=0.14 \pm 0.19 ; \mathrm{Z}_{\text {depolarizedBoSrev }}=1.47 \pm 0.29 ; p<0.01 ; n=$ 6 cells $(1$ of the 7 cells was depolarized strongly enough to inactivate spiking totally and thus is not included here; also note that only a significant effect on reverse BOS was observed but that the mean response to forward BOS more than doubled in the depolarized state).

brane potential $(0 / 8$ cells). Finally, in further contrast to $\mathrm{X}$-projecting neurons, tonic depolarization could unmask latent excitatory responses to reverse BOS in RA-projecting neurons (reverse BOS-evoked firing rate $>$ baseline; $p<0.05 ; 2 / 8$ cells at the normal membrane potential vs $4 / 8$ cells during tonic depolarization), and the mean firing-rate response strength to reverse BOS playback increased $>10$-fold with depolarization $\left(\mathrm{Z}_{\text {normalBosrev }}=\right.$ $0.14 \pm 0.19 ; Z_{\text {depolarizedBoSrev }}=1.47 \pm 0.29 ; p<0.01$, paired $t$ test). The contrasting effects of membrane polarity on song-evoked responses in X-and $\mathrm{RA}$-projecting neurons suggest that the same auditory stimuli drive different synaptic responses in the two $\mathrm{HVc}$ relay cell types; X-projecting neurons receive excitatory and inhibitory inputs activated primarily by forward BOS playback, whereas
RA-projecting neurons receive predominantly excitatory inputs activated both by forward and reverse BOS.

\section{The effects of membrane polarity on song selectivity}

Membrane potential manipulations differentially affected statistical measures of selectivity in the two relay cell classes, because of contrasting effects of membrane potential on firing-rate responses to forward and reverse BOS playback. Selectivity $\left(d^{\prime}\right)$ values calculated in both the normal and depolarized conditions revealed that the mean firing-rate selectivity for the population of X-projecting neurons, but not RA-projecting neurons, changed as a function of membrane polarity (Fig. 10). In $\mathrm{X}$-projecting neurons, manipulating membrane potential altered 


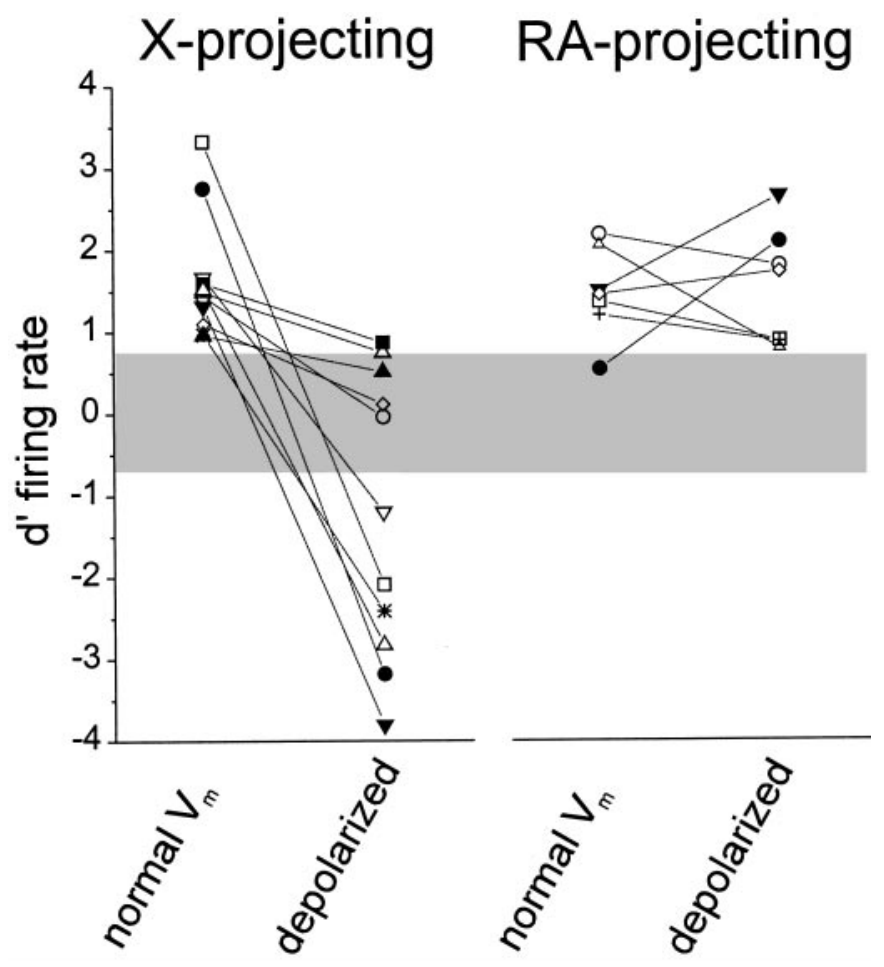

Figure 10. Altering the membrane potential at a single-cell level affected the firing-rate selectivity of X-projecting neurons but not that of RAprojecting neurons. Mean firing-rate selectivity $\left(d^{\prime}\right)$ for forward versus reverse BOS playback was measured for $11 \mathrm{X}$-projecting neurons and 7 RA-projecting neurons at their normal membrane potentials and then during tonic depolarization with positive current (X-projecting, $\mathrm{V}_{\text {control }}=$ $-76.5 \pm 2.1 \mathrm{mV}$ vs $\mathrm{V}_{\text {depolarized }}=-57.6 \pm 3.5 \mathrm{mV} ; p<0.001 ; n=11$ cells; RA-projecting, $\mathrm{V}_{\text {control }}=-79.6 \pm 2.8 \mathrm{mV}$ vs $\mathrm{V}_{\text {depolarized }}=-57.4 \pm 3.2$ $\mathrm{mV} ; p<0.0001 ; n=8$ cells). Left, In X-projecting neurons, this manipulation altered firing-rate selectivity $\left(d^{\prime}\right.$ normal $=1.65 \pm 0.22 \mathrm{vs} d^{\prime}{ }_{\text {depolar- }}$ ized $=-1.21 \pm 0.52 ; p<0.005$, paired $t$ test), because of changes in suprathreshold responses to forward, but not reverse, BOS playback (see Fig. 9B). Right, In RA-projecting neurons, tonic depolarization did not alter firing-rate selectivity $\left(d^{\prime}\right.$ control $=1.47 \pm 0.21 ; d^{\prime}{ }_{\text {depolarized }}=1.54 \pm 0.27 ; p=$ 0.85 , paired $t$ test), because of a parallel trend toward increased suprathreshold responsiveness to both forward and reverse BOS (see Fig. 9B). The horizontal gray bar is the nonselective zone.

mean firing-rate selectivity $\left(d^{\prime}=1.65 \pm 0.22 \mathrm{vs} d^{\prime}{ }_{\text {depolarized }}\right.$ $=-1.21 \pm 0.52 ; p<0.005$, paired $t$ test), whereas in RAprojecting neurons, mean firing-rate selectivity did not vary with membrane potential $\left(d^{\prime}{ }_{\text {normal }}=1.47 \pm 0.21 ; d^{\prime}{ }_{\text {depolarized }}=\right.$ $1.54 \pm 0.27 ; p=0.85$, paired $t$ test; membrane potential values in the two states are those given in the previous section and are in the legend for Fig. 10). On a single-cell level, membrane potential manipulations decreased the $d^{\prime}$ values of all $\mathrm{X}$-projecting neurons. At their normal membrane potentials, $11 / 11 \mathrm{X}$-projecting neurons had $d^{\prime}$ values $>0.7$, whereas in the tonically depolarized state, 9/11 cells had $d^{\prime}$ values $<0.7(6 / 9$ cells; $\left.d^{\prime}<-0.7\right)$, and the two other cells that maintained $d^{\prime}$ values $>0.7$ still exhibited lower $d^{\prime}$ values when depolarized. In contrast, the six RA-projecting neurons that were (positively) selective at their normal resting membrane potentials maintained selectivity during depolarization (the $d^{\prime}$ values decreased for 4 cells and increased for 2 cells, but all were $>0.7$ ), whereas a seventh cell that was initially nonselective exhibited selectivity during depolarization $\left(d^{\prime}{ }_{\text {normal }}=0.54 ; d^{\prime}{ }_{\text {depolarized }}=2.1\right)$. At a population level, these different effects on selectivity were caused by the distinctly different effects that depolarization had on firing rate to forward or reverse BOS playback, as noted previously (Figs. 8, 9). For X-projecting neurons, the specific membrane potential-dependent changes to forward but not reverse BOS responses resulted in altered selectivity, whereas for RA-projecting neurons, the parallel changes in forward and

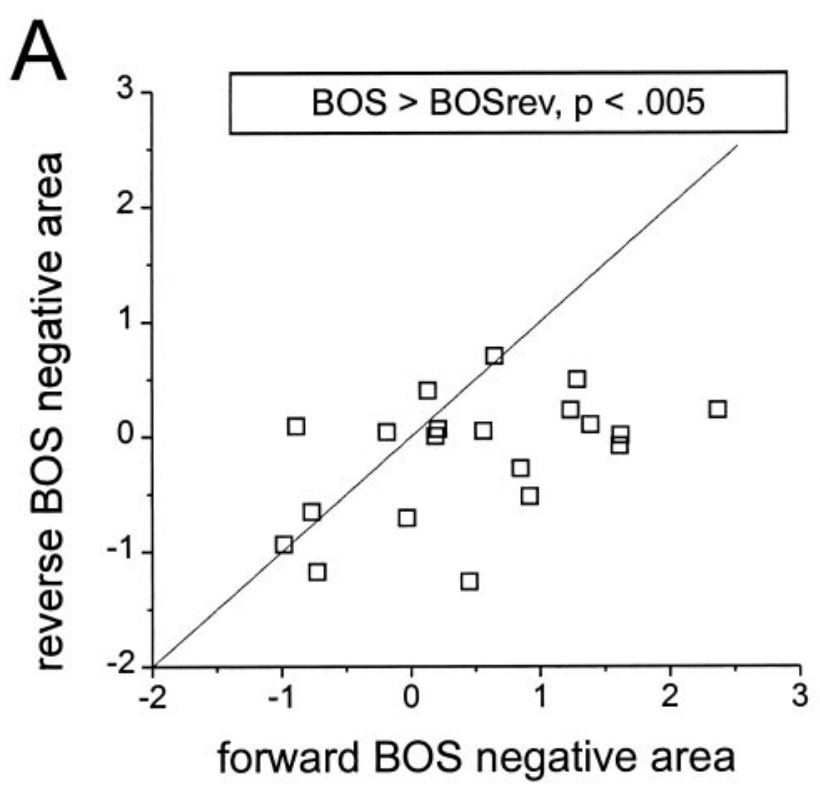

B

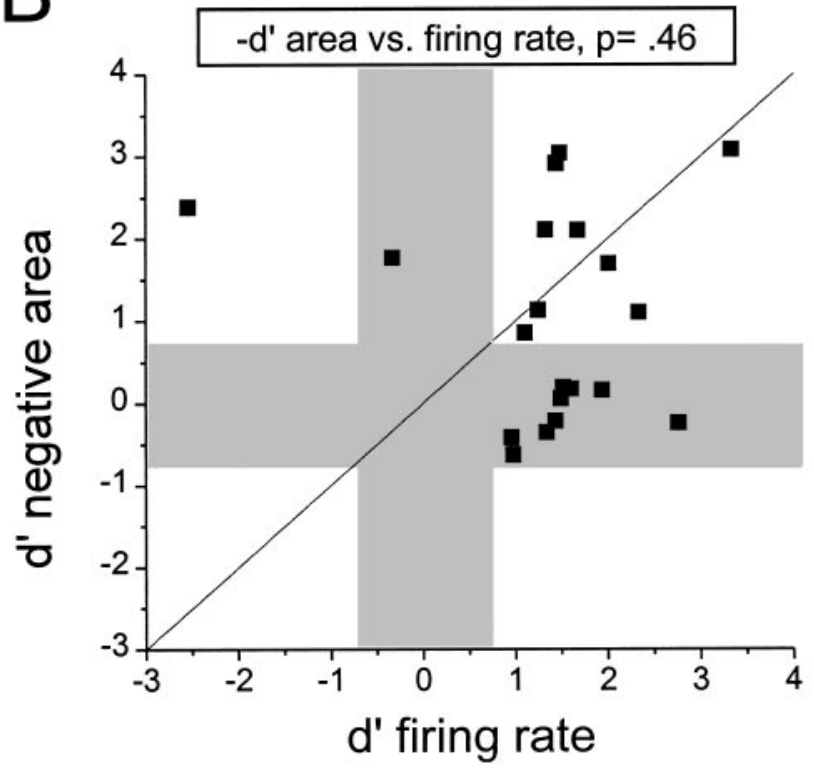

Figure 11. X-projecting $\mathrm{HVc}$ neurons exhibit inhibition that is strongly evoked by forward but not reverse BOS playback. $A$, A direct comparison of z-scores for subthreshold hyperpolarizations (net negative area, measured with respect to spontaneous hyperpolarizations in a $1.5 \mathrm{sec}$ window before the stimulus) evoked by forward ( $x$-axis) versus reverse ( $y$-axis) BOS playback is shown. Paired $t$ tests indicate that membrane hyperpolarization was more strongly evoked by forward BOS playback $(n=20$ cells; $p$ value shown above plot). $B$, Within-cell comparisons of subthreshold hyperpolarizing selectivity $\left(d^{\prime}\right.$ negative area) and firing-rate selectivity ( $d^{\prime}$ firing rate) for forward over reverse BOS playback show that the two measures are equally biased toward forward BOS playback $(n=20$ cells; $p$ value from paired $t$ test shown above plot). The unity line represents equivalent subthreshold and suprathreshold selectivity, whereas the gray bars demarcate zones of nonselective subthreshold (horizontal) or firing-rate (vertical) responses. Note that these relationships contrast with those for response strength and selectivity measured for depolarizing subthreshold responses and firing rate in X-projecting neurons (see Fig. 7).

reverse BOS responses resulted in equivalent selectivity at the two membrane potentials.

\section{Hyperpolarizing subthreshold and firing-rate responses in X-projecting neurons}

In tonically depolarized X-projecting neurons, the pronounced hyperpolarizations evoked by forward but not reverse BOS playback suggest that these cells receive highly song-selective inhibitory 
A
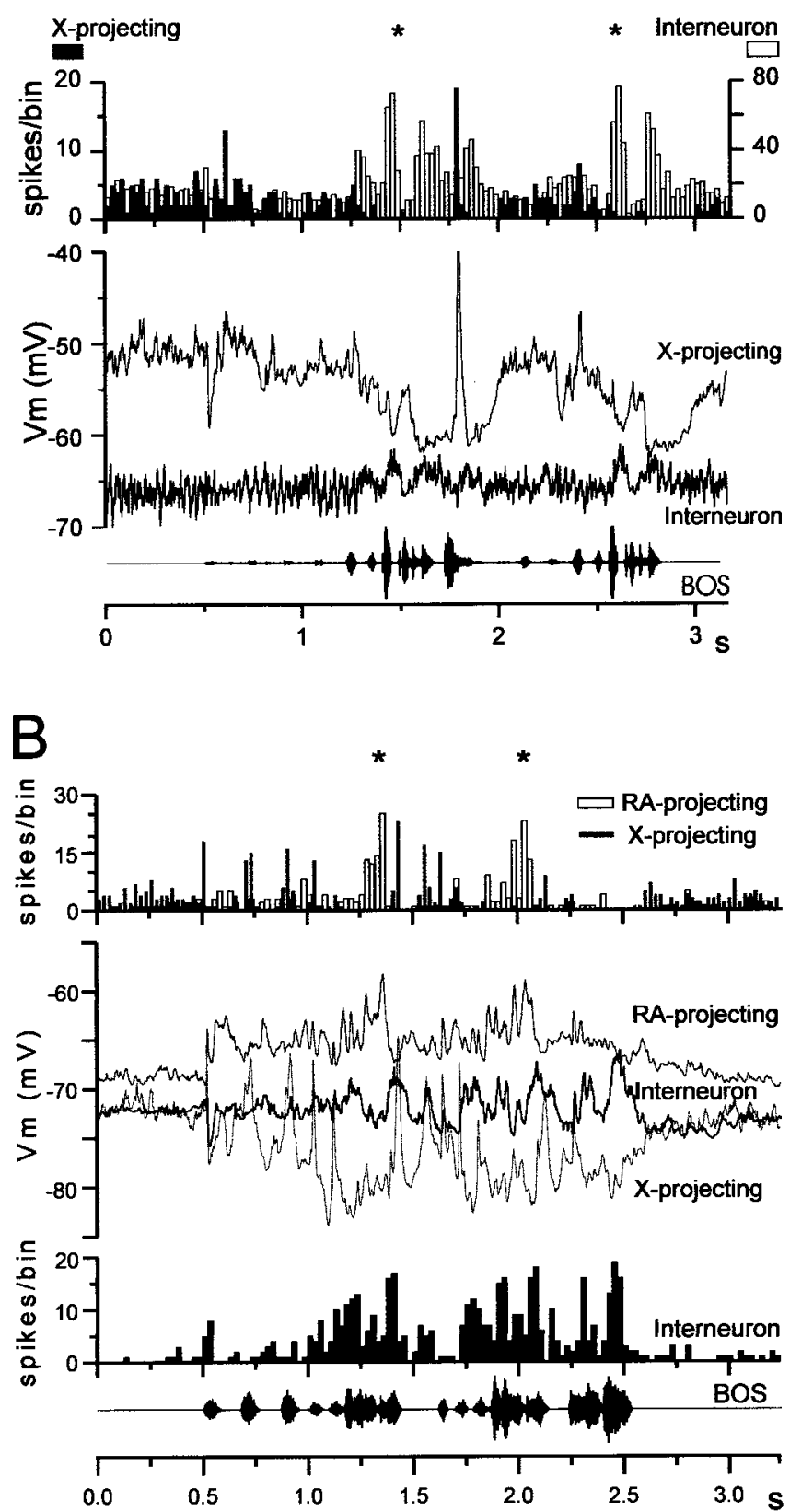

Figure 12. Within-bird comparisons of action potential responses and subthreshold responses in different $\mathrm{HVc}$ neuron types to forward BOS playback. $A$, The action potential PSTHs (top) and median-filtered averaged membrane potential responses (middle) aligned to forward BOS playback (oscillogram at bottom) of an X-projecting neuron and interneuron that were recorded sequentially from a single bird. Note that the periods of greatest firing-rate elevation in the interneuron (marked by asterisks) coincide with maximal firing-rate suppression and membrane hyperpolarization in the X-projecting neuron and that the subthreshold responses of the two cell types move in a mirror-symmetrical manner. $B$, The action potential PSTHs (top, second from bottom) and median-filtered averaged membrane potential responses (second from top) aligned to forward BOS playback of an X-projecting neuron, an RA-projecting neuron, and an interneuron that were recorded sequentially from a single bird. Note that (1) BOS-evoked firing of the RA-projecting neuron (marked by asterisks) alternates with firing of the X-projecting neuron, (2) the subthreshold responses of the two relay cells move in opposite directions relative to their resting potential (i.e., depolarizing for the RA-projecting cell and hyperpolarizing for the X-projecting cell), and (3) the song-evoked elevation in the interneuron firing closely coincides with when the RA-projecting cell is firing and when the period of maximal firing-rate suppression and membrane hyperpolarization occurs in the X-projecting neuron. PSTHs were generated using a $25 \mathrm{msec}$ bin width; all responses shown are to 20 iterations of the stimulus.

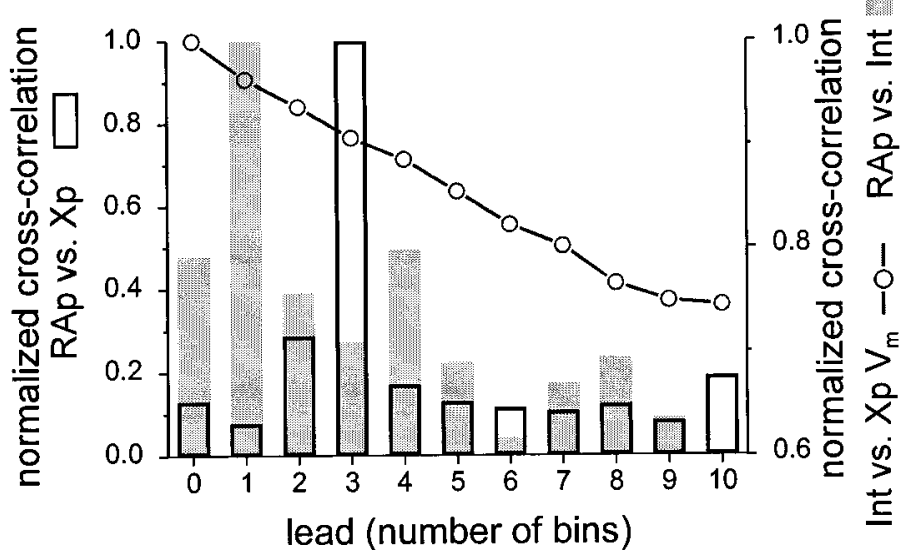

Figure 13. Cross-correlation analysis shows that, during BOS playback, peak interneuron firing closely coincides with the maximum hyperpolarization of X-projecting neurons, whereas the firing of RA-projecting and $\mathrm{X}$-projecting neurons is separated by long time intervals, and the firing of RA-projecting neurons and interneurons is separated by shorter intervals. The relative spike timing and/or membrane polarity during BOS playback was quantified by generating cross-correlograms from the action potential PSTHs of RA-projecting and X-projecting HVc neurons (open columns), the action potential PSTHs of RA-projecting neurons and interneurons (shaded columns), or the interneuron action potential PSTHs and $\mathrm{X}$-projecting neuronal membrane potential $\left(\mathrm{V}_{m}\right.$, averaged over $25 \mathrm{msec}$ intervals; joined circles). Cross-correlation values were generated from pairwise comparisons between two cells recorded sequentially within a single bird and then normalized to peak and averaged across all pairs of a given type (i.e., across birds; RA-projecting vs X-projecting, 6 pairs in 6 birds; RA-projecting vs interneurons, 4 pairs in 4 birds; interneurons vs $\mathrm{X}$-projecting $\mathrm{V}_{m}$, 6 pairs in 6 birds; see Materials and Methods). Time values are expressed in the bin widths of the PSTHs (i.e., $25 \mathrm{msec}$ ); note that the different scales of the two $y$-axes refer to different pairwise comparisons.

input. To explore this idea further, the relationship between subthreshold hyperpolarizing responses and firing-rate selectivity was examined for X-projecting neurons. First, as performed previously for depolarizing responses, subthreshold hyperpolarizing responses to forward and reverse BOS were compared using z-scores (Fig. 11A). These comparisons revealed that hyperpolarizing responses were biased toward forward BOS (mean z-score for hyperpolarization, BOS, $-0.49 \pm 0.21$; BOSrev, $0.16 \pm 0.12 ; p<0.005$, paired $t$ test). Second, direct comparisons of subthreshold hyperpolarizing selectivity ( $d^{\prime}$ for negative area) and firing-rate selectivity for individual X-projecting neurons were made (Fig. 11B). Unlike the mismatch between depolarizing subthreshold selectivity and firing-rate selectivity (see Fig. 6), hyperpolarizing subthreshold responses were as selective as firing-rate measures in these cells $\left(d^{\prime}\right.$ negative area $=1.05 \pm 0.28 ; d^{\prime}{ }_{\text {firing rate }}=1.35 \pm 0.26 ; p=0.46$, paired $t$ test). Taken together with the effects of membrane potential manipulations on forward (but not reverse) BOS-evoked hyperpolarizations, these results are consistent with the idea that $\mathrm{X}$-projecting neurons receive highly song-selective inhibition.

\section{Within-bird comparisons of the timing of song-evoked responses}

$\mathrm{HVc}$ interneurons are one possible source of the highly songselective inhibition seen in X-projecting neurons. To study this possibility further, sequential recordings from two or more different HVc neuron types were obtained within a single bird. Comparisons of the timing of forward BOS-evoked action potential firing and subthreshold responsiveness in these different cells were made by directly superimposing PSTHs and/or membrane potential traces collected from within birds (Fig. 12). In addition, crosscorrelation analyses were performed on cell pairs to examine during BOS playback the relative spike timing of RA-projecting neurons with that of either X-projecting neurons or interneurons and to compare interneuron spike timing with X-projecting neuro- 
nal membrane polarity (Fig. 13; Materials and Methods). These comparisons reveal that the timing of song-evoked responses varies systematically according to cell class, with the closest temporal coincidence between interneuron firing and X-projecting neuronal hyperpolarization. In six animals, recordings were obtained from at least one interneuron and one X-projecting neuron. In every case, song-evoked interneuron firing closely coincided with when hyperpolarization and firing-rate suppression occurred in the $\mathrm{X}$-projecting neuron (examples in Fig. 12). Cross-correlation analysis of interneuron firing and X-projecting neuronal membrane hyperpolarization during playback confirmed a maximum correlation at zero offset (Fig. 13; $n=6$ pairs in 6 birds). Although recordings obtained from both interneurons and $\mathrm{RA}$-projecting neurons revealed that song-evoked firing could occur near the same times (Fig. 12B, compare bottom, top PSTHs), cross-correlating their spike times revealed a slight offset, with RA-projecting neuronal firing leading that of interneurons by $\sim 25 \mathrm{msec}$ (the resolution of the analysis; see Fig. 13; $n=4$ pairs in 4 animals). Finally, recordings from the two relay cell types in single birds showed that they fired reciprocally during BOS playback and that the BOSevoked changes in membrane potential described an antiparallel motion, with the RA-projecting neuron exhibiting a prolonged depolarization during the same period when the X-projecting neuron was sustaining a net hyperpolarization (Fig. 12B). Crosscorrelation analysis confirmed a large temporal offset in songevoked firing between X-projecting and RA-projecting neurons, with a maximum of $\sim 75$ msec (Fig. $13 ; n=4$ pairs in 4 animals). These analyses indicate that, during forward BOS playback, interneuron firing closely coincides with $\mathrm{X}$-projecting neuronal hyperpolarization, RA-projecting neuron firing and interneuron firing are separated by short delays, and RA- and X-projecting neurons fire reciprocally at relatively long time intervals.

\section{DISCUSSION}

All HVc neuron types fire selectively for forward over reverse BOS playback. Relay cells innervating either area X or nucleus RA generate remarkably similar patterns of selective firing via different subthreshold mechanisms, likely because of segregated inhibitory input from interneurons onto X-projecting cells. Ultimately, factors that modulate the relay cells' membrane potentials may enable them to transmit distinct song representations, providing the potential for auditory comparisons used in song learning, maintenance, and perception.

Different morphological classes of HVc neurons examined in vivo display distinct intrinsic electrophysiological properties, extending previous in vitro studies (Dutar et al., 1998; Kubota and Taniguchi, 1998). One difference in vivo was that all cell types had lower input resistances than seen in brain slices, likely because of higher spontaneous synaptic activity in the intact brain. In vivo, as in vitro, positive currents drove interneurons to fire "fast" action potentials, often at high rates, while causing X-projecting neurons to fire regular action potential trains, although with a lower current-frequency relationship than for interneurons. Although RAprojecting neurons could fire repetitively in vivo, their DC-evoked spiking was characterized by an unusually prolonged and variable spike onset. Their firing remained intermittent and highly variable even with large currents $(\sim 1 \mathrm{nA})$, perhaps because of spontaneous synaptic activity interacting with injected currents. If these RAprojecting neurons are the same that become highly active during singing, then they undergo dramatic state-dependent modulation.

All three HVc neuron types are highly song selective. Previous intracellular studies concluded that $\mathrm{X}$ - but not RA-projecting neurons had auditory responses and left the auditory properties of HVc interneurons undetermined (Katz and Gurney, 1981; Lewicki, 1996). Here, interneurons fired tonically and selectively to BOS, whereas both relay cells fired more phasically. Such tonic responses, detected in previous intracellular studies (Lewicki, 1996), were not linked with a cell type; the present study shows they arise from $\mathrm{HVc}$ interneurons with fast spikes and varicose dendrites, two features of inhibitory interneurons in RA and in other systems (Azouz et al., 1997; Thomson and Deuchard, 1997; Spiro et al., 1999). If these HVc interneurons are also inhibitory, then they likely provide song-selective inhibition to X-projecting neurons. Previous reports classifying RA-projecting neurons as nonauditory (Katz and Gurney, 1981; Lewicki, 1996) are surprising because here they usually fired song-selectively and they always displayed robust song-selective subthreshold responses. Perhaps previous studies failed to detect auditory responses because song stimuli were not used (Katz and Gurney, 1981) or because these cells' relatively refractory behavior caused them to be disregarded.

The existence of song-responsive RA-projecting neurons, like those described here, has been inferred by reversibly inactivating $\mathrm{HVc}$, which transiently abolishes RA's auditory responsiveness (Doupe and Konishi, 1991). Furthermore RA's auditory responses persist even when LMAN (RA's other likely auditory afferent) is lesioned (Doupe and Konishi, 1991; Vicario and Yohay, 1993). A confound of understanding the genesis of RA's auditory responses in control birds is that inactivating $\mathrm{HVc}$ also silences X-projecting neurons, ultimately abolishing auditory responses in LMAN. Therefore, detecting auditory responses in RA-projecting neurons reveals a direct path from $\mathrm{HVc}$ to $\mathrm{RA}$ by which auditory information could influence vocalization. A parsimonious model is one in which the same RA-projecting $\mathrm{HVc}$ neurons that convey elaborate premotor patterns for singing (McCasland, 1987; Yu and Margoliash, 1996) also respond selectively to the resulting vocalizations, providing a cellular substrate for auditory feedback. Separate auditory and vocal projections from $\mathrm{HVc}$ to RA may be less likely to exist, because here RA-projecting neurons always responded to the BOS. In either case, HVc is the source of two song-selective auditory pathways.

Song-evoked subthreshold responses partly reflect the firing of neurons presynaptic to the impaled cell and thus can illuminate the origins of song selectivity. One caveat is that signal averaging measures net excitation and inhibition, and stronger elements may mask weaker components. Another qualification is that excitatory and inhibitory contributions may differ in states other than those studied here, perhaps via voltage-dependent effects (e.g., NMDA receptor-mediated currents). Nonetheless, one striking feature of RA-projecting neurons is that their song-evoked subthreshold responses appear exclusively (if latently) excitatory and, although biased toward forward song, can be quite pronounced to reverse BOS. The elevated and sustained firing in tonically depolarized RA-projecting neurons evoked by either stimulus (Fig. 8B, top) suggests that these cells receive little or no song-evoked inhibition. Instead, such sustained depolarizations indicate that cells presynaptic to RA-projecting neurons are excitatory and fire throughout forward and reverse BOS playback, although with a forward song bias. Most neurons in NIf, an HVc afferent, display sustained, song-selective firing (Janata and Margoliash, 1999), but the nature of their connections to different $\mathrm{HVc}$ neurons is unknown. In addition, a minority of neurons in the primary avian auditory telencephalon (field L) fire selectively to BOS (Lewicki and Arthur, 1996; Janata and Margoliash, 1999), but it is unknown whether they innervate HVc. A necessary step will be to determine the auditory nature and synaptic sign of extrinsic inputs onto various HVc neurons.

Unlike RA-projecting neurons, X-projecting neurons exhibited pronounced forward BOS-evoked hyperpolarizations and lack reverse BOS responses altogether. The contrasting song-evoked inhibition in the two relay cell types suggests that X-projecting neurons are privileged targets of $\mathrm{HVc}$ interneurons. Indeed, within birds, elevated interneuron firing closely coincides with $\mathrm{X}$-projecting neuronal hyperpolarization during forward BOS playback. In the songbird $\mathrm{HVc}$, BOS-evoked hyperpolarizations in a subset of X-projecting neurons may be critical to generating their supralinear sensitivity to syllable sequences (Lewicki and Konishi, 1995; Lewicki, 1996). Because such combination-sensitive neurons are rare $[\sim 15-25 \%$ of $\mathrm{HVc}$ neurons (Margoliash and Fortune, 1992; Lewicki and Arthur, 1996)], yet BOS-evoked hyperpolarizations were detected in all X-projecting neurons (at least when 
sufficiently depolarized), song-evoked inhibition alone may not suffice for this form of temporal sensitivity. Finally, the absence of reverse BOS responses in X-projecting cells contrasts directly with RA-projecting neurons, raising the possibility that the two relay cell types receive distinct extrinsic auditory inputs and/or form a serial or circular hierarchy in $\mathrm{HVc}$.

The function of BOS-evoked inhibition on X-projecting neurons could be to sculpt excitatory responses to BOS, generate more precise spike timing (Mainen and Sejnowski, 1995), elevate signalto-noise levels (Lewicki, 1996), or facilitate excitatory responses by either priming the postsynaptic membrane (e.g., by deinactivating voltage-gated conductances) or synchronizing the firing of excitatory presynaptic inputs. If X-and RA-projecting neurons receive similar song-selective excitatory inputs, then inhibition in $\mathrm{X}$-projecting neurons could partially suppress subthreshold depolarizing responses to forward BOS, causing them to appear nonselective. However, postsynaptic inhibition is unlikely to account for the lack of reverse BOS responses in X-projecting neurons, because hyperpolarizing responses to this stimulus were not detected even when cells were held tonically depolarized. Support for inhibitory maintenance of signal-to-noise levels and against postsynaptic priming comes from finding that artificially depolarizing $\mathrm{X}$-projecting neurons suppresses their mean firing below baseline during forward BOS playback, even though highly phasic firing persists (Fig. $8 A ; \sim 2.7 \mathrm{sec}$ ). Distinguishing between these various roles will require selectively blocking inhibition and assessing any effects on BOS-evoked responses in X-projecting neurons. Finally, these results underscore that highly similar action potential responses can arise from distinct subthreshold processes, even within a single brain area; both RA- and X-projecting neurons fired phasically and with almost identical song selectivity, yet only $\mathrm{X}$-projecting neurons displayed BOS-evoked hyperpolarizations.

Chronic recordings in nonsinging, awake zebra finches show that BOS playback evokes responses in HVc (Dave et al., 1998; but see Schmidt and Konishi, 1998), whereas after singing, HVc's auditory responsiveness is transiently suppressed (McCasland and Konishi, 1981). Because of HVc's functional heterogeneity, it will be necessary to distinguish whether auditory suppression also occurs during singing and in all $\mathrm{HVc}$ cell types and whether suppression varies in different developmental or attentional states, any of which could profoundly impact auditory-vocal interactions. One speculation is that the same circuitry used for singing is co-opted for the auditory processing of song. Specifically RA-projecting neurons could act via local interneurons to drive feedforward inhibition onto X-projecting cells both during singing and passive listening. In the singing state, vocal premotor-coupled inhibition may define a time window for auditory feedback, suppressing auditory responses when they fail to coincide closely with the vocal efference. During passive listening, auditory-evoked activity in RA-projecting neurons may again drive feedforward inhibition onto X-projecting neurons, shaping but not suppressing their responses. This model predicts that NIf neurons, which are likely to provide auditory and premotor drive to $\mathrm{HVc}$, innervate RA-projecting neurons, which in turn excite interneurons providing segregated input to $\mathrm{X}$-projecting neurons.

Dual auditory representations of song emerge from $\mathrm{HVc}$, potentially facilitating comparisons important to song learning and maintenance. The subthreshold events underlying BOS-evoked firing in the two relay cell types enable them to transmit different auditory representations of song, at least under certain conditions. When sufficiently depolarized, RA-projecting neurons fire continuously to forward BOS, and even to reverse BOS, normally a weak stimulus for HVc neurons (Margoliash, 1986; Lewicki and Arthur, 1996; Volman, 1996; Theunissen and Doupe, 1998). Therefore, the HVc-to-RA pathway may be able to convey information about song quality even when notes are distorted or sung out of sequence, as occurs in juveniles during song learning and in adults of those species that seasonally reexpress vocal plasticity. In contrast, inhibitory interactions may restrict X-projecting neuronal firing to certain times during the song, perhaps after distinct note sequences.
Coincident activation of the two pathways, perhaps when vocalizations include these specific sequences, could then reinforce motor patterns in $\mathrm{HVc}$ and/or in $\mathrm{RA}$, the direct and indirect target, respectively, of RA- and X-projecting cells. Furthermore X- and not RA-projecting neurons are likely sites for auditory refinement; varying the membrane potential of single X-projecting neurons altered their firing-rate selectivity, and as a whole these neurons displayed a marked increase in firing rate over subthreshold depolarizing selectivity. Because song selectivity arises via auditory experience (Volman, 1993), synapses onto X-projecting neurons may be especially sensitive to this experience. Future work can gauge the functional significance of multiple song representations, how HVc's local circuits refine song selectivity relative to HVc's extrinsic auditory afferents, and the sources of these extrinsic afferents.

\section{REFERENCES}

Alvarez-Buylla A, Theelen M, Nottebohm F (1988) Birth of projection neurons in the higher vocal center of the canary forebrain before, during, and after song learning. Proc Natl Acad Sci USA 85:8722-8726.

Azouz R, Gray C, Nowak L, McCormick D (1997) Physiological properties of inhibitory interneurons in cat striate cortex. Cereb Cortex 7:534-545.

Benton S, Cardin JA, Devoogd TJ (1998) Lucifer yellow filling of area $\mathrm{X}$-projecting neurons in the high vocal center of female canaries. Brain Res 799:138-147.

Bottjer SW, Miesner EA, Arnold AP (1984) Forebrain lesions disrupt development but not maintenance of song in passerine birds. Science 224:901-903.

Brainard MS, Doupe AJ (2000) Interruption of a basal ganglia-forebrain circuit prevents plasticity of learned vocalizations. Nature 404:762-766.

Dave AS, Yu AC, Margoliash D (1998) Behavioral state modulation of auditory activity in a vocal motor system. Science 282:2250-2254.

Doupe A, Kuhl P (1999) Birdsong and human speech: common themes and mechanisms. Annu Rev Neurosci 22:567-631.

Doupe AJ, Konishi M (1991) Song-selective auditory circuits in the vocal control system of the zebra finch. Proc Natl Acad Sci USA 88:11339-11343.

Dutar P, Vu HM, Perkel DJ (1998) Multiple cell types distinguished by physiological, pharmacological, and anatomic properties in nucleus Hvc of the adult zebra finch. J Neurophysiol 80:1828-1838.

Fortune ES, Margoliash D (1995) Parallel pathways and convergence onto $\mathrm{HVc}$ and adjacent neostriatum of adult zebra finches (Taeniopygia guttata). J Comp Neurol 360:413-441.

Foster EF, Bottjer SW (1998) Axonal connections of the high vocal center and surrounding cortical regions in juvenile and adult male zebra finches. J Comp Neurol 397:118-138.

Green D, Swets J (1966) Signal detection theory and psychophysics. New York: Wiley.

Jagadeesh B, Wheat HS, Kontsevich LL, Tyler CW, Ferster D (1997) Direction selectivity of synaptic potentials in simple cells of the cat visual cortex. J Neurophysiol 78:2772-2789.

Janata P, Margoliash D (1999) Gradual emergence of song selectivity in sensorimotor structures of the male zebra finch song system. J Neurosci 19:5108-5118.

Katz LC, Gurney ME (1981) Auditory responses in the zebra finch's motor system for song. Brain Res 221:192-197.

Kittelberger J, Mooney R (1999) Lesions of an avian forebrain nucleus that disrupt song development alter synaptic connectivity and transmission in the vocal premotor pathway. J Neurosci 19:9385-9398.

Kubota M, Taniguchi I (1998) Electrophysiological characteristics of classes of neuron in the HVc of the zebra finch. J Neurophysiol 80:914-923.

Lewicki MS (1996) Intracellular characterization of song-specific neurons in the zebra finch auditory forebrain. J Neurosci 16:5855-5863.

Lewicki MS, Arthur BJ (1996) Hierarchical organization of auditory temporal context sensitivity. J Neurosci 16:6987-6998.

Lewicki MS, Konishi M (1995) Mechanisms underlying the sensitivity of songbird forebrain neurons to temporal order. Proc Natl Acad Sci USA 92:5582-5586.

Mainen ZF, Sejnowski TJ (1995) Reliability of spike timing in neocortical neurons. Science 268:1503-1506.

Margoliash D (1983) Acoustic parameters underlying the responses of song-specific neurons in the white-crowned sparrow. J Neurosci 3:1039-1057.

Margoliash D (1986) Preference for autogenous song by auditory neurons in a song system nucleus of the white-crowned sparrow. J Neurosci 6:1643-1661.

Margoliash D, Fortune ES (1992) Temporal and harmonic combinationsensitive neurons in the zebra finch's HVc. J Neurosci 12:4309-4326.

McCasland JS (1987) Neuronal control of birdsong production. J Neurosci 7:23-39.

McCasland JS, Konishi M (1981) Interaction between auditory and motor 
activities in an avian song control nucleus. Proc Natl Acad Sci USA 78:7815-7819.

Nixdorf BE, Davis SS, DeVoogd TJ (1989) Morphology of Golgiimpregnated neurons in hyperstriatum ventralis, pares caudalis in adult male and female canaries. J Comp Neurol 284:337-349.

Nottebohm F, Stokes TM, Leonard CM (1976) Central control of song in the canary, Serinus canarius. J Comp Neurol 165:457-486.

Paton JA, O'Loughlin BE, Nottebohm F (1985) Cells born in adult canary forebrain are local interneurons. J Neurosci 5:3088-3093.

Rosen M, Mooney R (2000) Intrinsic and extrinsic contributions to auditory selectivity in a song nucleus critical for vocal plasticity. J Neurosc 20:XXXX-XXXX.

Scharff C, Nottebohm F (1991) A comparative study of the behavioral deficits following lesions of various parts of the zebra finch song system: implications for vocal learning. J Neurosci 11:2896-2913.

Scharff C, Nottebohm F, Cynx J (1998) Conspecific and heterospecific song discrimination in male zebra finches with lesions in the anterior forebrain pathway. J Neurobiol 36:81-90.

Schmidt MF, Konishi M (1998) Gating of auditory responses in the vocal control system of awake songbirds. Nat Neurosci 1:513-518.

Solis MM, Doupe AJ (1997) Anterior forebrain neurons develop selectivity by an intermediate stage of birdsong learning. $J$ Neurosci 17:6447-6462.

Spiro J, Dalva M, Mooney R (1999) Long-range inhibition within the zebra finch song nucleus RA can coordinate the firing of multiple projection neurons. J Neurophysiol 81:3007-3020.

Sutter ML, Margoliash D (1994) Global synchronous response to autogenous song in zebra finch HVc. J Neurophysiol 72:2105-2123.

Theunissen FE, Doupe AJ (1998) Temporal and spectral sensitivity of complex auditory neurons in the nucleus $\mathrm{HVc}$ of male zebra finches. J Neurosci 18:3786-3802.

Thomson A, Deuchard J (1997) Synaptic interactions in neocortical local circuits: dual intracellular recordings in vitro. Cereb Cortex 7:510-522.

Vates GE, Broome BM, Mello CV, Nottebohm F (1996) Auditory pathways of caudal telencephalon and their relation to the song system of adult male zebra finches. J Comp Neurol 366:613-642.

Vicario DS (1993) A new brain stem pathway for vocal control in the zebra finch song system. NeuroReport 4:983-986.

Vicario DS, Yohay KH (1993) Song-selective auditory input to a forebrain vocal control nucleus in the zebra finch. J Neurobiol 24:488-505.

Volman SF (1993) Development of neural selectivity for birdsong during vocal learning. J Neurosci 13:4737-4747.

Volman SF (1996) Quantitative assessment of song-selectivity in the zebra finch "high vocal center." J Comp Physiol [A] 178:849-862.

Wild JM (1993) Descending projections of the songbird nucleus robustus archistriatalis. J Comp Neurol 338:225-241.

Yu AC, Margoliash D (1996) Temporal hierarchical control of singing in birds. Science 273:1871-1875. 